04,13

\title{
Новый сценарий кинетики зарядки диэлектриков при облучении электронами средних энергий
}

\author{
() Э.И. Рау, А.А. Татаринцев \\ Московский государственный университет им. М.В. Ломоносова, \\ Москва, Россия \\ E-mail: rau@phys.msu.ru
}

Поступила в Редакцию 25 ноября 2020 г.

В окончательной редакции 25 ноября 2020 г.

Принята к публикации 25 ноября 2020 г.

На основе критического анализа предшествующих работ по исследованию механизмов зарядки диэлектрических мишеней под воздействием инжекции электронных пучков средних энергий $(1-30 \mathrm{keV})$ выявлено значительное число противоречивых данных в моделях зарядки, как теоретических, так и экспериментальных. Проведена ревизия и упорядочены причинно-следственные связи физического явления зарядки с целью устранения возникших противоречий в трактовке процессов электронной зарядки диэлектриков. После обширных экспериментальных исследований широкого класса диэлектриков установлены общие закономерности кинетики зарядки диэлектрических мишеней в зависимости от количества исходных и радиационно-индуцированных ловушек, плотности тока облучающих электронов $j_{0}$ и их энергии $E_{0}$. Показано, что вторично-эмиссионные свойства заряженного диэлектрика кардинально отличаются от незаряженного, а коэффициент эмиссии электронов $\sigma$ в зависимости от $E_{0}$ не является единственно определяющим фактором положительной или отрицательной зарядки. В рассмотрении процессов зарядки впервые включены первичные термализованные электроны, существенно меняющие общую картину зарядки, а также показана ключевая роль в кинетике зарядки плотности образующихся радиационных дефектов. В предложенной модели решающим стабилизирующим эффектом наступления равновесного состояния зарядки является генерируемое при облучении внутреннее электрическое поле $F_{\text {dip }}$ между положительно и отрицательно заряженными слоями в приповерхностной области диэлектрика. Главной движущей силой саморегулирующегося самосогласующегося процесса зарядки диэлектриков при электронном облучении выступает не только коэффициент эмиссии электронов, как общепринято считалось ранее, а формирование электрического поля дипольного слоя зарядов. Это критическое регулирующее поле $F_{\text {cr }}$ порядка $0.5 \mathrm{MV} / \mathrm{cm}$ приблизительно одинаково для всех диэлектриков при любых значениях $E_{0}$.

Ключевые слова: зарядка диэлектриков, радиационное дефектообразование, равновесное состояние зарядки, вторичная электронная эмиссия.

DOI: 10.21883/FTT.2021.04.50713.246

\section{1. Введение}

Интерес к радиационной физике диэлектриков со временем все возрастает. Особую важность представляет физика взаимодействия электронных пучков с диэлектрическими мишенями, в частности явления неизбежной электрической зарядки диэлектриков и их пробоев. Развитие теоретических и экспериментальных работ в области радиационной физики становится все более актуальной проблемой, так как имеет важное значение в различных областях науки и техники, например в электротехники, микроэлектронике, ядерной энергетике, ионно- и электронно-зондовых методах анализа поверхности, космической технике и многих других технологических процессах. Интересно мнение по этому поводу нобелевского лауреата Schein L. [1], отмечающего, что хотя явление электрической зарядки диэлектриков известно довольно давно, но является сюрпризом, что оно до сих пор является не до конца понятым и изученным в области физики твердого тела.
Актуальность исследований эффектов зарядки диэлектриков под воздействия облучения заряженными частицами диктуется и другими обстоятельствами. Так, например, по данным японского космического агентства [2] до недавнего времени причиной около 50\% катастрофических отказов в функционировании всех космических аппаратов является электростатические пробои между различно заряженными участками поверхности космических аппаратов из-за воздействия облучения космическими частицами [3]. Далее, зарядка диэлектрических образцов является критическим фактором в применении ионных и электронных методов исследования поверхности твердого тела, таких как электронная микроскопия, электронная и ионная литография, аналитические методы Оже-спектроскопии, вторичноионной масс-спектрометрии, локального рентгеновского микроанализа и т.д.

Существуют различные модели электризации диэлектрических мишеней при электронном облучении, но ни одну из них, особенно более ранних, нельзя принять 
исчерпывающей, дающей полное представление о сложном самосогласующимся механизме эмиссии вторичных электронов, о частичном захвате на энергетические ловушки первичных электронов, образовании внутренних электростатических полей и генерацию дополнительных радиационных дефектов. Вначале считалось, что краеугольным моментом процесса зарядки диэлектрических мишеней является значение полного коэффициента вторичной электронной эмиссии $\sigma[4,5]$. При равенстве $\sigma=1$ образец не заряжается, при $\sigma>1$ образец заряжается положительно, при $\sigma<1-$ отрицательно. Но со временем эта концепция устарела, так как множество экспериментов не согласовывалось с указанной стандартной моделью зарядки. Основополагающая идея о решающей роли вторично-электронной эмиссии оказалась не вполне состоятельной для заряжающихся при облучении диэлектрических мишеней.

Следующим существенным шагом в прояснении эффектов зарядки явилась разработка модели биполярно заряженных слоев [6,7], согласно которой при электронном облучении электрически непроводящих образцов образуются тонкий приповерхностный слой положительного заряда за счет ухода вторичных электронов, и нижележащий более толстый слой отрицательного заряда за счет аккумулируемой на ловушках части первичных электронов. Теория этого механизма зарядки нашла экспериментальное подтверждение во многих работах (см. например [8,9]). Дальнейшее развитие этой модели с существенными уточнениями и дополнениями получило в теории электронной зарядки диэлектриков на основе рассмотрения баланса токовых потоков заряженных частиц в облучаемом объеме мишени и эмиссионного тока вторичных и отраженных электронов [10-12]. Но и здесь анализ оказался в ряде важных моментов ошибочным, резко контрастирующим с данными многих экспериментальных работ. Основным разногласием между результатами работ $[8,9]$ и $[11,12]$ явилось сильное расхождение времен зарядки диэлектриков (на порядки величин!). Причины принципиальных ошибок и ограничений, а также разногласий будут рассмотрены в следующем разделе настоящей работы.

\section{2. Критический анализ существующих моделей зарядки облучаемых диэлектриков}

Общепринятые в течение долгого времени представления о механизме зарядки массивных диэлектриков под воздействием облучения электронными с энергиями $E_{0}$ от сотен $\mathrm{eV}$ до десятков $\mathrm{keV}$ основаны на классической теории вторичной электронной эмиссии $[4,5,13,14]$. При бомбардировке образцов электронным пучком с силой тока $I_{0}$ часть электронов $I_{0} \eta_{0}$ отражается от мишени, суммируясь с током вторичных электронов $I_{0} \delta_{0}$. Здесь введем обозначение $\sigma_{0}=\eta_{0}+\delta_{0}$, где индекс ,0“ означает принятые значения для незаряженного диэлект-

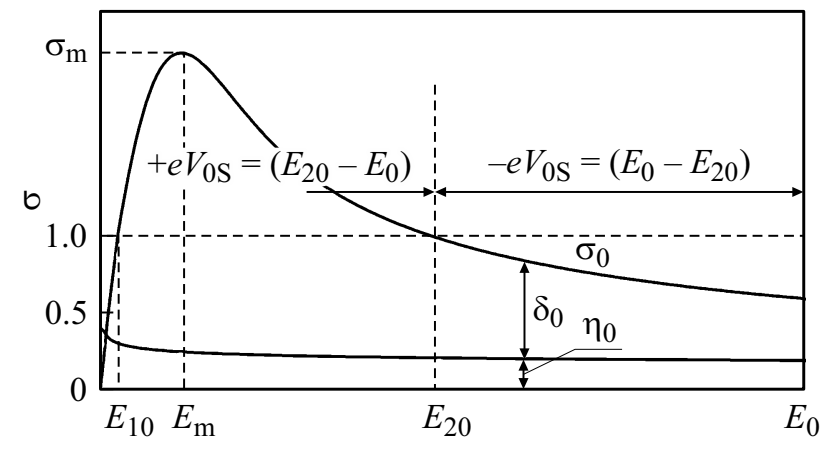

Рис. 1. Качественная зависимость коэффициента полной электронной эмиссии $\sigma_{0}$ и коэффициента отраженных электронов $\eta_{0}$ от их энергии $E_{0}$ для незаряженных диэлектриков.

рика. Коэффициент отраженных электронов $\eta_{0}$ слабо зависит от энергии $E_{0}$ и для многих диэлектриков $0.1 \leq \eta_{0} \leq 0.2$. Коэффициент вторичной электронной эмиссии $\delta_{0}=f\left(E_{0}\right)$ с хорошим приближением выражается соотношениями $[5,7]$ :

$$
\begin{gathered}
\delta_{0}=\frac{B E_{0} \lambda_{0}}{E_{i} R_{0}}\left[1-\exp \left(\frac{-R_{0}}{\lambda_{0}}\right)\right] \\
\frac{\delta_{0}}{\delta_{m}}=1.28\left(\frac{E_{m}}{E_{0}}\right)^{0.67}\left[1-\exp \left(-1.61\left(\frac{E_{0}}{E_{m}}\right)^{1.67}\right)\right],
\end{gathered}
$$

где $B-$ вероятность преодоления вторичными электронами (ВЭ) поверхностного потенциального барьера, $E_{i} \approx 3 E_{g}+1$ - это энергия генерации электроннодырочных пар, пропорциональная ширине запрещенной зоны $E_{g}, R_{0}$ - глубина пробега первичных электронов в материале мишени, $\lambda_{0}$ - глубина выхода ВЭ, $\delta_{m}-$ максимальное значение коэффициента ВЭ при энергии $E_{0}=E_{m}$. Зависимость $\sigma_{0}=\eta_{0}+\delta_{0}$ от энергии $E_{0}$ качественно представлена на рис. 1.

Если образец электрически не проводящий, то при энергиях первичных электронов $E_{0}$, больших некоторой критической энергии $E_{20}$ (вторая критическая энергия), имеем $\sigma_{0}<1$. В этом случае часть электронов $\left(1-\eta_{0}\right)$ имплантируется в мишень, заряжая ее отрицательно до равновесного потенциала $-V_{\mathrm{S} 0}=\frac{\left(E_{0}-E_{20}\right)}{q}$, где $q-$ элементарный заряд электрона. Если энергия $E_{0}$ меньше $E_{20}$, но больше первой кроссоверной энергии $E_{10}$, то $\sigma_{0}>1$ и тогда формируется положительный поверхностный потенциал $+V_{\mathrm{S}}=\frac{\left(E_{20}-E_{0}\right)}{q}$. Процесс зарядки саморегулируется таким образом, что при отрицательной зарядке реальная энергия первичных электронов при достижении поверхности под воздействием отрицательного зарядового потенциала образца $-V_{\mathrm{S}}$ уменьшается от начального значения $E_{0}$ до равновесной величины

$$
E_{L 0}=E_{0}-q V_{\mathrm{S} 0}=E_{20}=\text { const. }
$$

Считалось, что в интервале энергий $E_{10}<E_{0}<E_{20}$ образец заряжается положительно и соответственно 
сдвигает энергию $E_{0}$ в сторону ее увеличения до равновесной энергии $E_{L}=E_{20}$. При кроссоверных равновесных энергиях $E_{0}=E_{10}$ и $E_{0}=E_{20}$ имеем $\sigma_{0}=1$ и поэтому диэлектрик не заряжается. Как будет показано далее в настоящей работе, эти рассуждения справедливы только для идеального гипотетического случая, когда диэлектрик при электронном облучении не заряжается, что практически невыполнимо.

В действительности, как показывают наши многочисленные эксперименты, во-первых, форма и характер кривой эмиссии электронов, представленной на рис. 1, существенно отличаются при электронной зарядке диэлектриков. Во-вторых, энергия $E_{20}$ не является константой, но зависит от $E_{0}$. Далее, при $E_{0}=E_{20}$ образец, как правило, заряжается отрицательно, а в интервале энергий $E_{10}<E_{0} \leq E_{20}$ все диэлектрики заряжаются не до потенциала $+V_{\mathrm{S}}=\frac{\left(E_{L}-E_{20}\right)}{q}$, а лишь до единиц (максимум десятков) вольт. Равновесное значение $E_{L 0}$ может достигаться с отклонениями от $E_{20}$ на доли и даже единицы $\mathrm{keV}$.

По изложенной выше стандартной модели зарядки поверхностный потенциал выражается следующим соотношением $[13,14]$ :

$$
V_{\mathrm{S}}(t)=\frac{\left(E_{0}-E_{20}\right) k I_{0} R}{1+k I_{0} R}\left[1-\exp \left(-\frac{\left(1+k I_{0} R\right) h}{\varepsilon_{r} \varepsilon_{0} R A} t\right)\right],
$$

где $R$ - сопротивление образца толщиной $h, \varepsilon_{r}$ - относительная диэлектрическая проницаемость диэлектрика, $\varepsilon_{0}$ - абсолютная диэлектрическая константа вакуума, $A$ - площадь облучаемого участка поверхности образца, $t$ - время облучения, $k-$ коэффициент, выражающий наклон кривой $\sigma_{0}\left(E_{0}\right)$ в точке $E_{20}\left(k=\frac{1-\sigma_{0}}{E_{0}-E_{20}}\right)$. В начальные моменты времени облучения

$$
t_{1} \ll \frac{\varepsilon_{r} \varepsilon_{0} R A}{\left(1+k I_{0} R\right) h}=\tau
$$

кинетика зарядки описывается, согласно (3), выражением

$$
V_{\mathrm{S}}(t)=\left(E_{0}-E_{20}\right) \frac{k I_{0} d t}{\varepsilon_{r} \varepsilon_{0} A}=\frac{\left(1-\sigma_{0}\right) I_{0} h}{\varepsilon_{r} \varepsilon_{0} A} t .
$$

При достижении равновесного состояния через время $\tau$ :

$$
V_{\mathrm{S} 0}=\left(E_{0}-E_{20}\right) \frac{k I_{0} R}{1+k I_{0} R}=\frac{I_{0} R(1-\sigma)\left(E_{0}-E_{20}\right)}{\left(E_{0}-E_{20}\right)+I_{0} R(1-\sigma)} .
$$

Время равновесной зарядки диэлектриков, приводимое в работе [14], равно

$$
\tau_{0} \approx \frac{\varepsilon_{r} \varepsilon_{0} a E_{20}}{q I_{0}(1-\eta)}
$$

где $a-$ линейный размер облучаемой площади. Оценки при $I_{0}=10^{-9} \mathrm{~A}, A=a^{2}=10^{-2} \mathrm{~cm}^{2}, \eta_{0}=0.2$, $\varepsilon_{r}=10, E_{0}=15 \mathrm{keV}, E_{20}=5 \mathrm{keV}$ приводят к значению $\tau_{0}=10^{-2} \mathrm{~s}$. Но при указанных параметрах, близких в

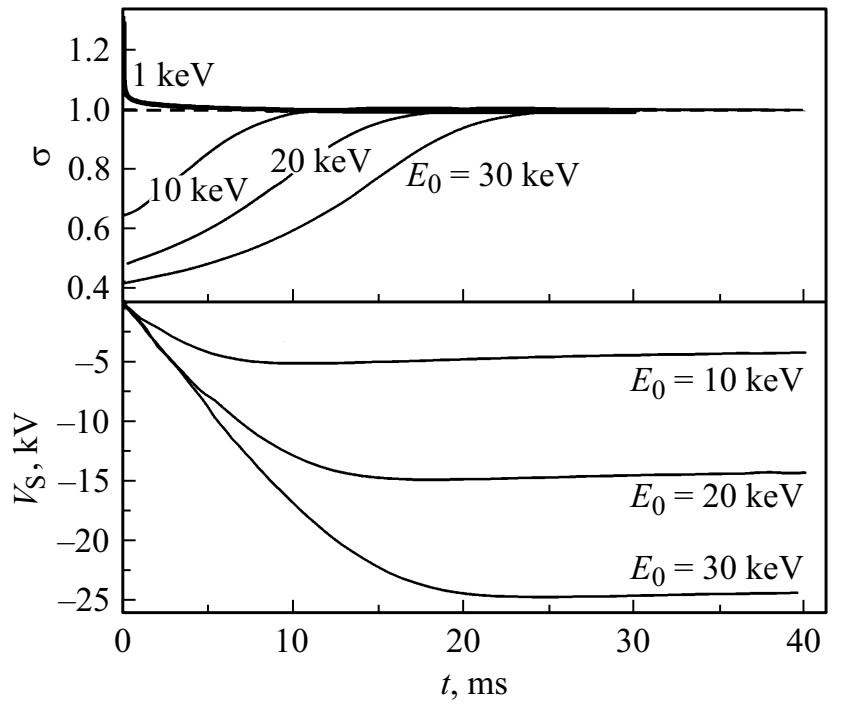

Рис. 2. Расчетные зависимости коэффициент эмиссии электронов $\sigma$ (вверху) и поверхностного потенциала $V_{\mathrm{S}}$ (внизу) образца из оксида алюминия в зависимости от времени облучения $t$ для различных энергий электронов пучка $E_{0}$ при плотности тока $j_{0}=10^{-5} \mathrm{~A} / \mathrm{cm}^{2}$. Графики взяты из работ $[11,12]$.

наших экспериментах к случаю облучения $\mathrm{Al}_{2} \mathrm{O}_{3}$ (сапфир), различие между расчетным по формуле (7) и экспериментальным значениями $\tau_{0}$ достигает порядков величин (не доли секунд, а сотни секунд соответственно - сравни далее рис. 2 и рис. 5 ).

Чтобы устранить или частично сгладить большие противоречия изложенной стандартной модели зарядки, в работах [6-8] предложена модель двухслойных приповерхностных зарядов, нашедшая в своей основе обширное экспериментальное подтверждение в работax $[8,9,15,16]$. Согласно последней теории при облучении диэлектрических мишеней электронами в приповерхностной области образуется тонкий слой положительного заряда толщиной $\lambda_{0}$ из-за ухода вторичных электронов. Если плотность тока первичных электронов равна $j_{0}$, то поверхностная плотность $\sigma^{+}$положительного заряда равна

$$
\sigma^{+}=j_{0} \int_{0}^{t} \delta(t) d t=\lambda_{0} \rho_{+}
$$

где $\rho_{+}$- объемная плотность положительных зарядов. Одновременно с образованием положительного слоя часть первичных электронов $j_{0}\left(1-\eta_{0}\right)$ аккумулируется в толще диэлектрика в слое, равном глубине пробега $R_{0}$, с поверхностной плотностью $\sigma^{-}$:

$$
\sigma^{-}=j_{0} \int_{0}^{t}\left(1-\eta_{0}\right) d t=R_{0} \rho_{-},
$$

где $\rho_{-}$- объемная плотность отрицательных зарядов. Под воздействием этого биполярного распределения 
суммарный заряд $\Delta Q=\left|Q_{-}-Q_{+}\right|$электризует поверхность до равновесного потенциала

$$
V_{\mathrm{S} 0}\left(1+K^{\prime}\right)=\frac{(\sigma-1) \Delta Q h}{\varepsilon_{r} \varepsilon_{0} A}-\frac{\left(\Delta Q^{+} \lambda+\left(1-\eta_{0}\right) Q^{-} R_{0}\right)}{2 \varepsilon_{r} \varepsilon_{0} A},
$$

где $h$ - толщина диэлектрической мишени, $A$ площадь облучения, $K^{\prime}=h / \varepsilon_{r} L, L-$ расстояние до плоскости коллектора (спектрометра). Перепад абсолютных значений потенциалов $\Delta V_{S}$ между эффективной плоскостью положительных зарядов $\left(\approx \lambda_{0} / 2\right)$ и плоскостью на глубине $R_{0} / 2$ (центр тяжести отрицательных зарядов) образует поле $F_{\text {in }}=\frac{2 \Delta V_{S}}{R_{0}}$, разносящее генерируемые электроны и дырки в противоположные стороны внутри объема генерации. Ток этих носителей является радиационно-стимулированным током $I_{R}$, определяемым выражением [8]:

$$
I_{R}=\frac{2 \Delta V_{\mathrm{S}}}{R_{0}-\lambda_{0}} A \gamma ; \gamma=\gamma_{0} D^{0.5}=\gamma_{0}\left(\frac{10^{8} I_{0} E_{0}}{A R_{0} \rho}\right)^{0.5} .
$$

Здесь в выражение для удельной проводимости $\gamma[\Omega \cdot \mathrm{m}]^{-1}$ входит значение дозы облучения $D$, $\gamma_{0}$ - калибровочная доза $(1 \mathrm{rad} / \mathrm{s}), \rho$ - здесь плотность материала мишени $\left[\mathrm{g} / \mathrm{cm}^{3}\right]$. Заметим, что ток $I_{R}$ не влияет на абсолютную величину поверхностного потенциала $V_{\mathrm{S}}$, так как в равной мере частично компенсирует как положительный, так и отрицательный заряд, т.е. величина $\Delta Q=\left|Q_{-}+Q_{+}\right|$не изменяется. Но и в этой прогрессивной модели имеются свои неопределенности и недостатки. Основные из них заключаются в разногласиях расчетов по основной формуле (10) с экспериментальными результатами. Из экспериментов следует, что равновесный потенциал заряженной мишени $V_{\mathrm{S} 0}$ зависит от толщины образца $h$ только при условии, что $R_{0}$ по порядку величины равна $h$. При $h \gg R_{0} V_{\mathrm{S}}$ не зависит от $h$. Далее, вопреки выражению (10) в равновесном состоянии потенциал поверхности $V_{\mathrm{S} 0}$ слабо зависит от $\varepsilon_{r}$, что будет показано в настоящей работе.

Прямое измерение потенциала $V_{\mathrm{S}}\left(t, E_{0}, j_{0}\right)$ представляло определенные трудности, поэтому широкое распространение получил косвенный метод - расчет $V_{\mathrm{S}}$ по измеряемому аккумулируемому заряду $Q_{\mathrm{tr}}$. Этот заряд определяется либо при регистрации тока смещения $I_{d}$, наводимого на металлическую подложку образца, либо при измерении тока эмиссии $I_{\sigma}[17-23]$. Действительно, при зарядке поверхностного слоя мишени на близлежащем металлическом электроде (подложка образца) электрически индуцируются заряды $Q_{i}$ противоположного знака, что вызывает ток смещения $I_{d}$ относительно земли

$$
Q_{i}=-K Q_{\mathrm{tr}} ; \quad Q_{\mathrm{tr}}=-\frac{1}{K} \int_{0}^{t} I_{d}(t) d t
$$

где $K=\frac{L}{\left(L+h / \varepsilon_{r}\right)} \quad[20], \quad Q_{\mathrm{tr}}-$ абсолютная величина аккумулированного заряда, захваченного на ловушки.
Этим методом получено много полезной информации о кинетике зарядки диэлектриков. Однако в экспериментах возникают большие трудности при точном определении тока смещения $I_{d}$, т.к. одновременно на сигнальном электроде-подложке регистрируется омический ток утечки $I_{L}$. Этот недостаток значительно смягчается при независимой регистрации токов $I_{d}$ и $I_{L}$ на раздельных электродах [16], хотя паразитное взаимовлияние этих токов в регистрирующих цепях вряд ли удается полностью исключить. В результате аддитивности измеряемых токов $I_{d}+I_{L}$ получаются неверные результаты в определении $Q_{\mathrm{tr}}$ по формуле (12), а тем самым и завышенные значения поверхностного потенциала $V_{\mathrm{S}}$, определяемого, как правило, по соотношению для равномерно заряжен-

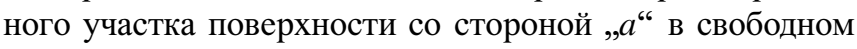
пространстве

$$
V_{\mathrm{S}}=\frac{\sigma_{\mathrm{tr}} h}{\varepsilon_{0}\left(\varepsilon_{r}+1\right)}=\frac{Q_{\mathrm{tr}}}{\varepsilon_{0}\left(\varepsilon_{r}+1\right) a},
$$

где $\sigma_{\mathrm{tr}}-$ поверхностная плотность аккумулированных зарядов. Аналогично, ошибки возникают при расчете $V_{\mathrm{S}}$ по измеренным потокам $I_{\sigma}=I_{0} \sigma_{\text {eff }}\left(\sigma_{\text {eff }}=\delta+\eta\right)$, когда аккумулируемый заряд $Q_{\mathrm{tr}}$ берется как разность $\left(I_{0}-I_{\sigma}\right) t$. В этих измерениях получаются заниженные значения $V_{\mathrm{S}}$, так как в $I_{\sigma} t$ входит доля зарядов $Q_{\mathrm{PT}}$ от первичных термализованных электронов, не вносящих вклад в зарядку. Отсюда еще раз вытекает необходимость прямого измерения поверхностного потенциала $V_{\mathrm{S}}$ по сдвигу спектров ВЭ, сопровождаемого одновременным измерением токов $I_{\sigma}$ и токов $I_{d}+I_{L}$. Именно этот комплексный прием позволил нам выявить все противоречивые сведения предыдущих работ и предложить новую модель зарядки диэлектрических мишеней под воздействием электронного облучения, изложенных в последующих разделах статьи.

В последние годы широко распространилась теория, основанная на решении транспортных уравнений заряженных частиц в диэлектрике и на законах сохранения баланса токов и зарядов $[11,12,24-28]$. Суть этой теоретической модели основана на трех базисных уравнениях: уравнение Пуассона, уравнение непрерывности тока носителей заряда и уравнение сохранения баланса зарядов. Уравнение Пуассона записывается в виде

$$
\begin{aligned}
\nabla^{2} V_{\mathrm{S}}(x, t) & =\frac{d F(x, t)}{d x}=\frac{\rho_{+-}(x, t)}{\varepsilon_{0} \varepsilon_{r}} \\
& =\frac{q\left[p_{\mathrm{tr}}+p-n_{\mathrm{tr}}-n\right]}{\varepsilon_{0} \varepsilon_{r}}
\end{aligned}
$$

где $\rho_{+-}-$объемная плотность (концентрация) свободных электронов и дырок $(n$ и $p)$ и захваченных на ловушечные центры электронов $n_{\mathrm{tr}}$ и дырок $p_{\mathrm{tr}}$ соответственно, $F=\frac{\sigma_{\mathrm{tr}}}{\varepsilon_{0} \varepsilon_{r}}-$ возникающее на поверхности мишени поле зарядов с эффективной поверхностной плотностью $\sigma_{\mathrm{tr}}$. 
Закон сохранения токовых потоков выражается соотношением

$$
I_{0}=\sigma_{\mathrm{SEB}} I_{0}+\frac{d Q_{\mathrm{tr}}}{d t}+I_{L},
$$

где $\sigma_{\mathrm{SEB}}=\delta+\eta-$ полный коэффициент эмиссии вторичных и отраженных электронов, $I_{L}$ - ток объемной и поверхностной утечки свободных носителей заряда. И, наконец, третье транспортное уравнение, отражающее сохранение баланса зарядов при плотности тока $J_{n}$ :

$$
\frac{\partial\left[n(x, t)+n_{\mathrm{tr}}(x, t)\right]}{\partial t}=\frac{1}{q} \nabla J_{n}(x, t)-r n(x, t) h(x, t),
$$

где $r$ - коэффициент рекомбинации электронов с плотностью $n(x, t)$ и дырок с плотностью $h(x, t)$.

$$
\begin{gathered}
J_{n}(x, t)=-q \mu_{e} n(x, t) \nabla V_{\mathrm{S}}(x, t)+q D_{e} \nabla n(x, t), \\
\frac{\partial n_{\mathrm{tr}}(x, t)}{\partial t}=n(x, t) \mu_{e} F(x, t) S_{e}\left[N_{i}-n_{\mathrm{tr}}(x, t)\right] \\
-n_{\mathrm{tr}}(x, t) \exp \left[-\frac{E_{T}-\Delta E_{\mathrm{PF}}}{k T}\right],
\end{gathered}
$$

где $N_{i}$ - плотность исходных электронных ловушек, $S_{e}$ - сечение захвата электронов, $E_{T}$ - энергия активации ловушки, $\Delta E_{\mathrm{PF}}-$ понижение энергетического уровня ловушки под действием поля $F_{\text {in }}$ (эффект Poole-Frenkel). Значение $\Delta E_{\mathrm{PF}}$ например для $\mathrm{Al}_{2} \mathrm{O}_{3}$, приводимое в [11], равно

$$
\begin{aligned}
\Delta E_{\mathrm{PF}}[\mathrm{eV}] & =10^{4}\left(\frac{q}{\pi \varepsilon_{0} \varepsilon_{r}}\right)^{1 / 2} F_{\text {in }}^{1 / 2}[\mathrm{MV} / \mathrm{cm}] \\
& =0.24 F_{\mathrm{in}}^{1 / 2}[\mathrm{MV} / \mathrm{cm}] .
\end{aligned}
$$

Аналогичные уравнения записываются для транспорта дырок $p$.

Самым серьезным изъяном этой, в общем-то цельной теоретической модели является катастрофическое различие вычисленных времен зарядки диэлектриков от измеренных во многих экспериментах. Так, расчетные времена зарядки, например монокристаллов окислов $\mathrm{SiO}_{2}$ и $\mathrm{Al}_{2} \mathrm{O}_{3}$, приведенные в работах $[11,12]$ (см. рис. 2), различаются от экспериментальных (см. например, [8,9,29-31]) на порядки величины: от десятков миллисекунд до десятков и сотен секунд соответственно. Коренной ошибкой работ [10-12] является тот факт, что в них кинетика зарядки оценивалась по временным характеристикам эмиссии электронов, в то время как значение равновесного потенциала измерялось по сдвигу края спектра тормозного рентгеновского излучения. Но время набора этого спектра составляет десятки секунд и никак не коррелирует со временем зарядки образца. Считалось, что есть полное временное соответствие между наступлением равновесного потенциала $V_{\mathrm{S} 0}$ и достижением коэффициента эмиссии электронов значения $\sigma=1$. Это не соответствует истине, на что было впервые обращено внимание в наших работах [9,31], а также в публикациях [16,32]. Причины указанного большого разногласия будут подробно обсуждаться ниже в настоящей работе.

\section{3. Экспериментальные результаты}

Эксперименты по изучению процессов зарядки диэлектрических образцов под воздействием электронного облучения проводились нами на сканирующем электронном микроскопе (СЭM) LEO-1455VP в рабочем вакууме $5 \cdot 10^{-6}$ Torr. СЭМ является весьма эффективным прибором для таких исследований, так как позволяет с одной стороны облучать мишени с контролируемой дозой в широком диапазоне энергий электронов (от сотен $\mathrm{eV}$ до десятков $\mathrm{keV}$ ), и с другой стороны проводить измерения характеристических параметров зарядки: значения эмиссии электронов, величины аккумулируемых зарядов и поверхностных потенциалов. Как показали наши эксперименты, для исключения значительных неопределенностей при регистрации кинетики процессов зарядки, указанные параметры необходимо измерять одновременно, что снимает ряд методических противоречий, наблюдаемых во многих предыдущих публикациях.

Схема нашей экспериментальной установки была подробно описана в более ранних работах $[15,29,30]$, поэтому здесь отметим лишь основные детали устройства (рис. 3). При исследованиях всех диэлектриков регистрировались ток полной эмиссии электронов $I_{\sigma}$, ток первичных электронов $I_{0}$, ток смещения, индуцированный на проводящей подложке образцов $I_{d}$, ток утечки $I_{L}$, а также поверхностный потенциал $V_{\mathrm{S}}$. Последний измерялся по временному сдвигу спектра ВЭ,

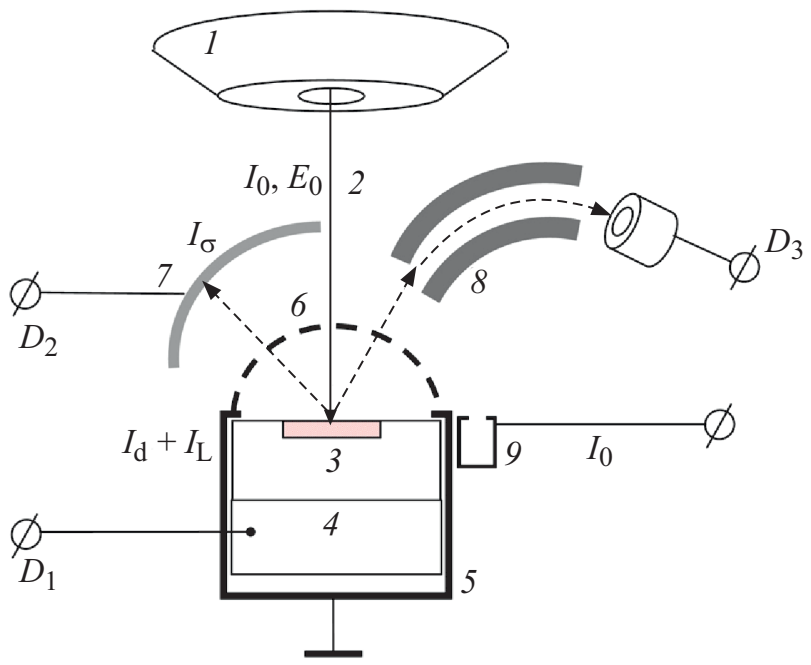

Рис. 3. Схема экспериментальной установки: 1 - объективна линза СЭМ, 2 - электронный зонд, 3 - диэлектрическая мишень, 4 - металлическая подложка образца, 5 - экранирующий корпус, $6-$ полусферическая сетка, $7-$ усеченный полусферический коллектор электронов, $8-$ сектор тороидального электронного спектрометра, 9 - цилиндр Фарадея. 
Основные физические параметры для исследованных диэлектриков

\begin{tabular}{c|c|c|c|c|c|c}
\hline Образец & $\rho, \mathrm{g} / \mathrm{cm}^{3}$ & $\varepsilon_{r}$ & $\delta_{m}\left(E_{m}\right)$ & $\beta, \Omega^{-1} \cdot \mathrm{cm}^{-1}$ & $E_{20}, \mathrm{keV}$ & $E_{2 \mathrm{C}}, \mathrm{keV}$ \\
\hline $\mathrm{SiO}_{2}$ & 2.6 & 3.9 & $3.2(0.5)$ & $10^{-10}$ & 4.4 & 1.25 \\
$\mathrm{Al}_{2} \mathrm{O}_{3}$ & 3.9 & 9.8 & $7.2(0.85)$ & $10^{-13}$ & 8.5 & 2.6 \\
Слюда & 2.6 & 6.0 & $2.3(0.4)$ & $10^{-13}$ & 2.0 & 3.2 \\
Тефлон & 2.2 & 2.0 & $2.4(0.3)$ & $10^{-14}$ & 1.75 & 2.5 \\
ПММА & 1.2 & 2.6 & $4.5(0.5)$ & $10^{-13}$ & 4.6 & 2.7
\end{tabular}

снимаемого в процессе зарядки с помощью тороидального электростатического спектрометра, специально адаптированного к СЭМ $[15,16,29,30]$. Типичные спектры $N(E)$ эмиттированных электронов, снятые в процессе облучения $\mathrm{Al}_{2} \mathrm{O}_{3}$-керамики, представлены в качестве примера на рис. 4, $а$ для случая положительной зарядки и на рис. $4, b$ для отрицательной зарядки мишени. Погрешность измерения высоковольтных потенциалов по положению спектральных пиков составляет не более 5\%, время регистрации одного спектра равно 3-5 s.
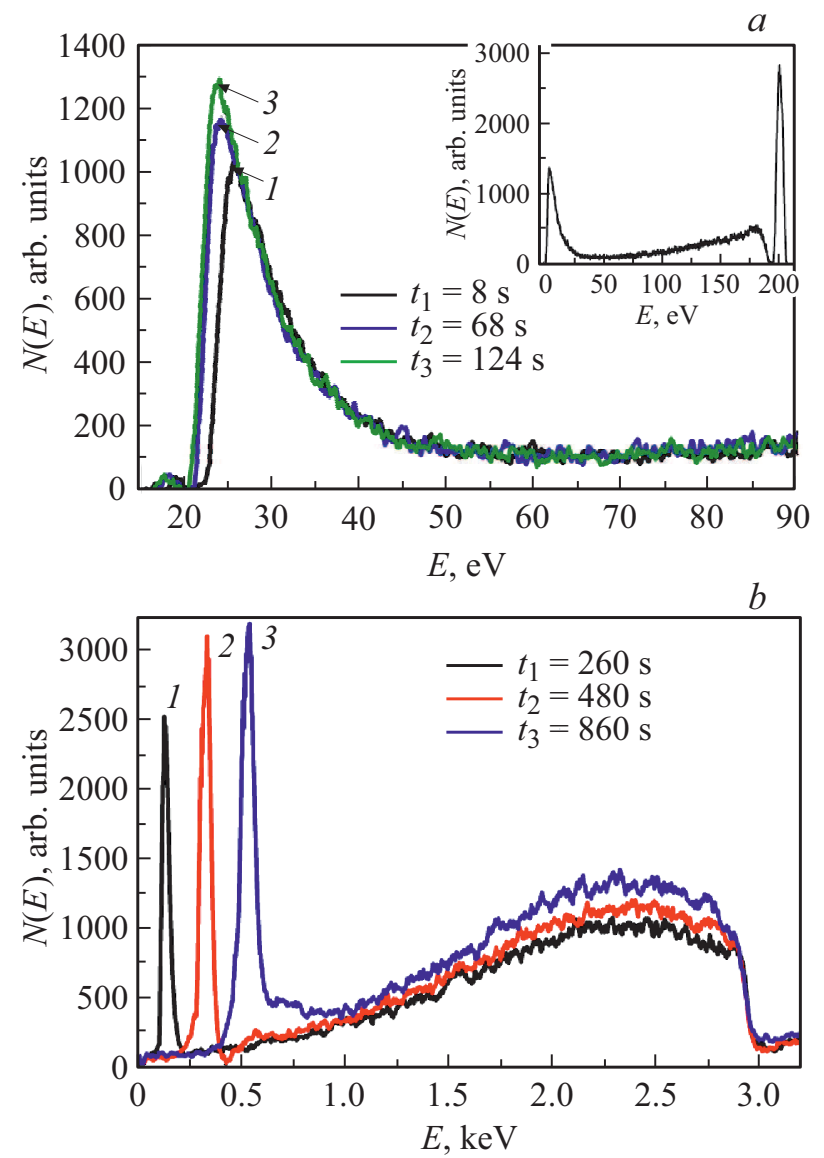

Рис. 4. Сдвиги спектров ВЭ в процессе зарядки $\mathrm{Al}_{2} \mathrm{O}_{3}$-керамики для двух значений энергии падающего электронного пучка: (a) $E_{0}=200 \mathrm{eV}$ (положительная зарядка) и (b) $E_{0}=3 \mathrm{keV}$ (отрицательная зарядка). Время положения соответствующих пиков ВЭ показаны на рисунке. На вставке (a) показан полный спектр эмиттированных электронов при $E_{0}=200 \mathrm{eV}$.

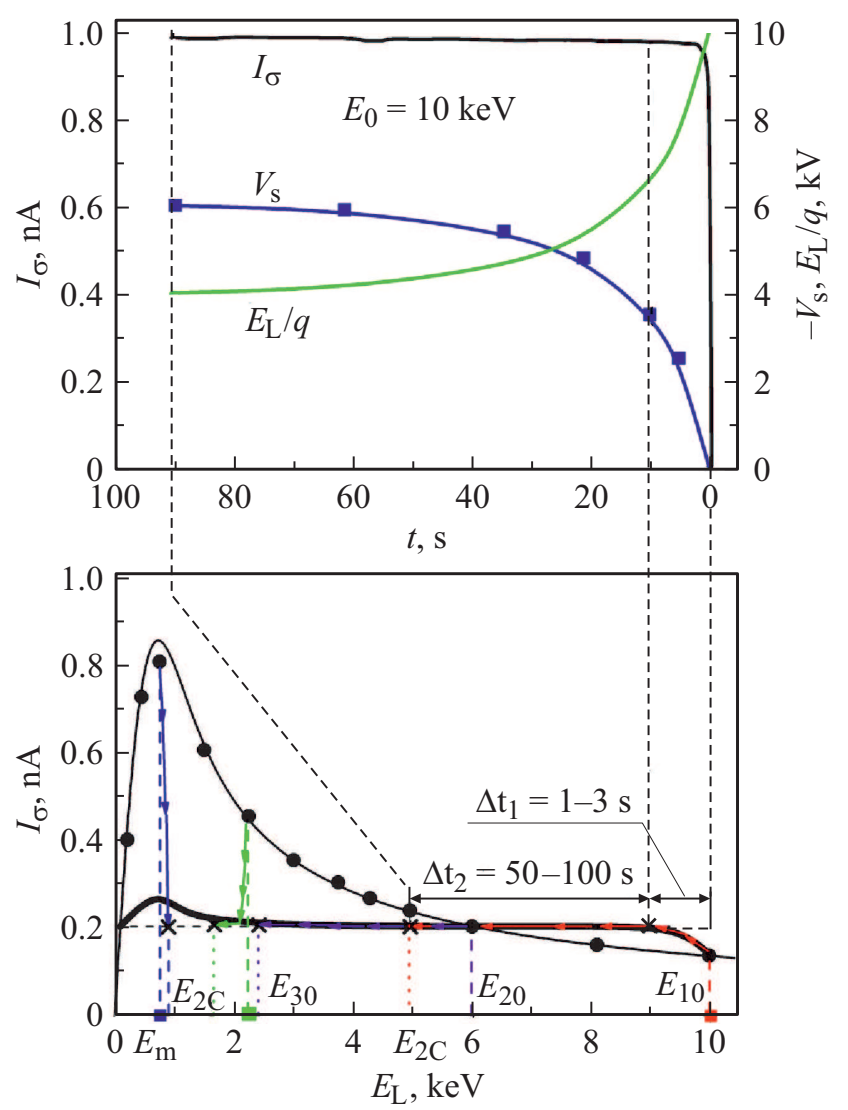

Рис. 5. Временнб́е характеристики зарядки диэлектриков на примере $\mathrm{Al}_{2} \mathrm{O}_{3}$-керамики при $E_{0}=10 \mathrm{keV}$.

Исследования проводились для широкого класса диэлектриков, отличающихся как по составу, так и по своим физическим параметрам. Основные значения этих параметров для диэлектриков, исследуемых в данной статье, представлены в таблице.

Результаты одновременных измерений тока эмиссии электронов $I_{\sigma}$ и поверхностных потенциалов $V_{\mathrm{S}}$ в зависимости от времени облучения $\mathrm{Al}_{2} \mathrm{O}_{3}$-керамики при $E_{0}=10 \mathrm{keV}, I_{0}=1 \mathrm{nA}, A=100 \times 100 \mu \mathrm{m}^{2}$, $j_{0}=10^{-5} \mathrm{~A} / \mathrm{cm}^{2}$ представлены на рис. 5 вверху, где также приведена временная зависимость текущей фактической энергии облучающих электронов $E_{L}(t)=E_{0}-q V_{\mathrm{S}}(t)=10 \mathrm{keV}-q V_{\mathrm{S}}(t)$. Примечательным является тот факт (в общих чертах характерный для всех диэлектриков!), что время установления 
стационарного равновесного состояния $\sigma=1$ намного меньше времени наступления равновесного состояния для зарядового потенциала поверхности $V_{\mathrm{S}}$. На этот неожиданный эффект нами было обращено внимание уже в ранних работах. Его игнорирование привело к неверной интерпретации кинетики зарядки диэлектрических мишеней в значительном числе публикаций (см. например $[11,12,14])$.

Интересно сопоставить график временной зависимости эмиссионных характеристик $I_{\sigma}$ заряжающегося образца при переменном значении эффективной энергии падающих электронов $E_{L}(t)$, представленных на рис. 5 вверху (штриховая кривая) со значениями $I_{\sigma}$ для случая незаряжающегося образца (сплошная кривая с экспериментальными точками) на рис. 5 внизу. Последняя зависимость взята из усредненных значений, снятых при малых дозах импульсного облучения мишени. Проведем более подробный анализ закономерностей, представленных на рис. 5. В данном примере при исходной стартовой энергии электронов $E_{0}=10 \mathrm{keV}$ уже в первый момент облучения $\Delta t_{1}=1-3 \mathrm{~s}$ эмиссионная характеристика достигает своего равновесного значения $\sigma \approx 1$, в то время как потенциал поверхности $V_{\mathrm{S}}$ еще далек от равновесного значения $V_{\mathrm{S} 0}$ и равен всего сотням вольт. Далее, по мере перманентной зарядки за гораздо более длительное время $\Delta t_{2}=50-100$ s коэффициент $\sigma$ остается равным единице $( \pm 0.01)$ в то время как потенциал $V_{\mathrm{S}}(t)$ за это длительное время плавно нарастает до значения равновесия $V_{\mathrm{S} 0}=7 \mathrm{keV}$, уменьшая эффективную энергию облучающего пучка электронов до $E_{L}=3 \mathrm{keV}$. Это значение отличается от равновесного значения энергии $E_{L} \neq E_{20}=6 \mathrm{keV}$ для случая незаряжающегося диэлектрика (где $\sigma_{0}=1$ на графике рис. 5) [4,5]. Приведенный пример является еще одним свидетельством кардинального различия эмиссионных характеристик заряженных и не заряженных диэлектриков при электронном облучении.

В принципе, равновесная „кроссоверная“ энергия для заряженного диэлектрика $E_{L}=E_{2 C}$ может быть как равной кроссоверной энергии $E_{20}$ для случая незаряженного диэлектрика (где $\sigma=1$ ), так и большей $E_{20}$. Эта ситуация обусловлена либо наличием поверхностных и объемных токов утечки свободных электронов либо, что более вероятно, наличием сильного внутреннего поля $F_{\text {in }}$, что будет рассмотрено в следующем разделе настоящей статьи. Но более закономерен случай, установленный нами экспериментально и предсказанный теоретически в работах [6,7], когда равновесная энергия при зарядке $E_{2 \mathrm{C}}$ меньше равновесной кроссоверной энергии $E_{20}$ для незаряженного диэлектрика (см. рис. 5). Так, если для оксидов металлов, как правило, $E_{20}>3 \mathrm{keV}$, то для них же $E_{2 \mathrm{C}}$ находится в районе $E_{2 \mathrm{C}} \approx 1 \mathrm{keV}$. Одним из регулирующих механизмов установления более низкого значения $E_{2 \mathrm{C}}$ по сравнению с $E_{20}$ является критическое поле $F_{\mathrm{dip}}=F_{\mathrm{cr}} \approx \frac{\Delta V_{ \pm}}{R_{0} / 2-\lambda / 2}$, возникающее между положительно и отрицательно заряженными слоями в при- поверхностной области мишени. В приведенном соотношении в числителе $\Delta V_{ \pm}=V_{-}-V_{+}$, а в знаменателе расстояние между центрами тяжести поверхностной плотности зарядов разных знаков. При этом следует учитывать, что если средняя глубина выхода ВЭ (эффективная толщина положительного слоя) мало изменяется в процессе зарядки, то средняя эффективная глубина слоя отрицательного заряда $R_{0}\left(E_{L}\right)$ уменьшается пропорционально уменьшению $E_{L}$.

Рассмотрим теперь случай, когда $E_{0}=E_{20}$, соответствующий для незаряженного диэлектрика равенству $\sigma=1$. Как правило, в начальные моменты облучения электронами с энергией $E_{20}>1 \mathrm{keV}$ глубина аккумуляции отрицательных зарядов $R\left(E_{20}\right)>\lambda$ и объем диэлектрика, способный захватить часть первичных электронов на ловушки, значительно больше объема формирования положительного слоя толщиной $\lambda$. Поэтому общий отрицательный заряд $Q_{-}$в объеме облучения больше, чем положительный заряд $Q_{+}$, а значит суммарный заряд $\Delta Q=\left|Q_{-}-Q_{+}\right|$отрицателен, что вызывает эффективный отрицательный потенциал поверхности. Мало того, этот отрицательный потенциал $-V_{\mathrm{S}}$ формируется не только при кроссоверной энергии $E_{0}=E_{20}$, но и в диапазоне энергий $2 E_{m}<E_{0}<E_{20}$. Причина та же: $R_{0}>\lambda_{\mathrm{SE}}$ вплоть до $R_{0}\left(E_{0}\right) \approx \lambda_{\mathrm{SE}}$, где потенциал $V_{\mathrm{S}}$ равен нулю. При $R_{0}<\lambda_{\mathrm{SE}}$, что выполняется в области энергий $E_{10}<E_{0}<2 E_{m}$ плотность положительных зарядов больше плотности отрицательных зарядов, и в этом диапазоне поверхностный потенциал положителен, достигая максимума при энергии $E_{0}=E_{m}$. Но положительный потенциал $+V_{\mathrm{S}}$ во всем диапазоне энергий $E_{10}<E_{0}<2 E_{m}$ не превышает по абсолютной величине значений единиц (максимум до десятков) вольт, так как при положительном потенциале поверхности часть эмиттированных ВЭ возвращается полем обратно на поверхность, частично компенсируя положительный заряд, и уменьшая эффективный коэффициент вторичной эмиссии. Выходу ВЭ препятствует и внутреннее электрическое поле в области положительного слоя зарядов, уменьшая глубину выхода $\lambda_{\mathrm{SE}}$, а также эффект частичной рекомбинации внутренних ВЭ с генерируемыми дырками (положительный заряд) или образование поляронов в слое толщиной $\lambda_{\mathrm{SE}}$. Какой из механизмов уменьшения тока эмиссии электронов в области положительной зарядки мишени (см. рис. 5 внизу) является доминирующим, требует специальных исследований. Фактом остается то обстоятельство, что при положительной зарядке фактическая энергия облучающих электронов $E_{L}$ почти не изменяется и не движется до точки $E_{20}$, как было принято ранее $[5,11,14]$. Эта энергия изменяется от $E_{0}($ сотни $\mathrm{eV})$ в сторону возрастания всего лишь на единицы (десятки) eV. В области $2 E_{m} \leq E_{0}<E_{20}$ энергия $E_{L}$ даже падает (вследствие отрицательной зарядки), причем значительно, достигая в ряде случаев единиц keV.

Аналогичные характеристики по величине и по временам зарядки имеют такие исследованные нами образцы, 

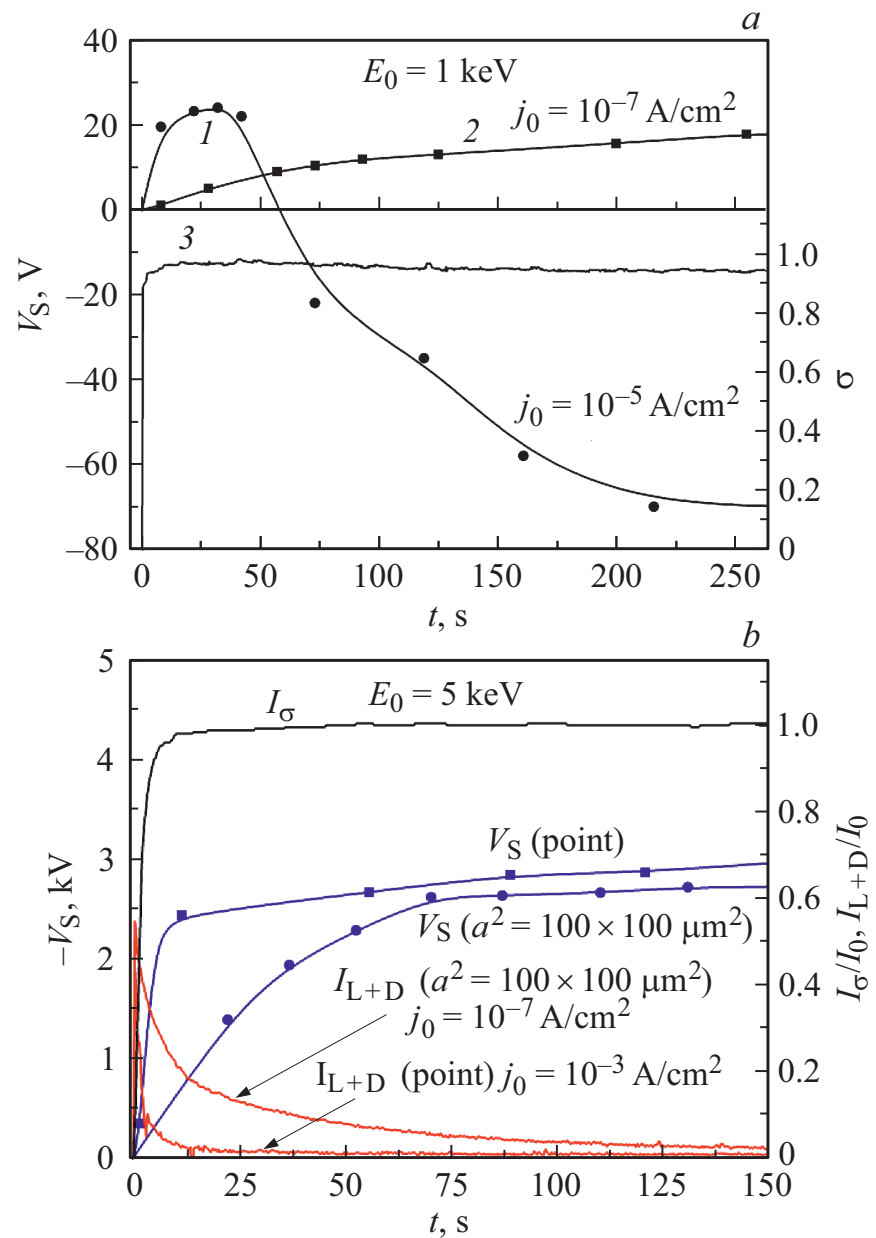

Рис. 6. Временнб́е характеристики параметров зарядки тефлона: коэффициента эмиссии электронов $\sigma(t)$, поверхностного потенциала $V_{\mathrm{S}}(t)$ и тока смещения и утечки $I_{\mathrm{L}+\mathrm{D}}(t)$ для двух значениях энергии первичного пучка электронов $E_{0}=1 \mathrm{keV}(a)$ и $E_{0}=5 \mathrm{keV}(b)$ и разных плотностях тока зонда.

как поликристаллический $\mathrm{Al}_{2} \mathrm{O}_{3}$, монокристаллы $\mathrm{SiO}_{2}$, кристаллы $\mathrm{NaCl}$, шпинель, рутил, слюда, ряд сегнетоэлектриков. В то же время полимерные диэлектрики (полиметилметакрилат, тефлон, антрацен, лавсан, полиэтилен) заряжается до схожих значений равновесных потенциалов $V_{\mathrm{S} 0}$, но за более короткие времена облучения, то есть при меньших дозах облучения первичными электронами. В качестве примера на рис. 6, a приводятся зарядовые характеристики тефлона $\left(\mathrm{CF}_{2}\right)$ типичные в той или иной мере всем полимерным материалам. Результаты измерений показывают аномальную зависимость потенциалов зарядки от плотности тока облучения при $E_{0}=1 \mathrm{keV}$. Ожидаемая положительная зарядка происходит только при сравнительно низких плотностях тока $\left(j_{0}=10^{-7} \mathrm{~A} / \mathrm{cm}^{2}\right)$, в то время как при высоких плотностях $\left(j_{0}=10^{-5} \mathrm{~A} / \mathrm{cm}^{2}\right)$ после начального положительного значения $+V_{\mathrm{S}}$ через $50 \mathrm{c}$ происходит инверсия зарядки и образец далее заряжается отрицательно. Ток эмиссии в обоих случаях приходит в равновесное значение гораздо быстрее, чем насыщение потенциала (через 1-2s).

На рис. 6, $b$ приводятся эмиссионные характеристики $I_{\sigma}$, а также временные зависимости поверхностного потенциала $-V_{\mathrm{S}}(t)$ и тока смещения $I_{d}(t)$, снятые на тефлоне при энергии пучка $E_{0}=5 \mathrm{keV}$ и плотности тока $j_{0}=10^{-6} \mathrm{~A} / \mathrm{cm}^{2}$ (площадь сканирования $100 \times 100 \mu \mathrm{m}^{2}$, $I_{0}=0.1 \mathrm{nA}$ ) и в режиме неподвижного зонда (точка) с плотностью тока облучающих электронов $10^{-3} \mathrm{~A} / \mathrm{cm}^{2}$. Здесь очевидно, что, несмотря на различные времена зарядки, зависящие от плотности тока, равновесный потенциал достигает почти одинакового значения $-3 \mathrm{kV}$. Интересно отметить, что расчеты методом МонтеКарло [33] предсказывают слабую положительную зарядку полимеров в начальные моменты облучения электронами даже при энергиях $E_{0}=10 \mathrm{keV}$. Авторы затруднились интерпретировать полученный результат. На наш взгляд это можно объяснить тем, что исходно в изучаемом образце имеется малое число дефектов, поэтому лишь незначительная часть имплантированных электронов захватывается на ловушки. В результате большее число электронов эмиттируются, чем аккумулируются в мишени, что обусловливает доминирование положительного заряда (положительного потенциала) в начальные моменты времени облучения. В дальнейшем процессе электронного облучения полимеров инициируется рождение радиационных дефектов (разрывы химических связей), способных захватывать свободные электроны и тем самым заряжать мишень до высоких отрицательных потенциалов [34]. Аналогичные характеристики присущи всем диэлектрикам в начальные моменты облучения относительно слабым потоком электронов с поверхностной плотностью $Q_{0} A^{-1}$ и дозой $Q_{0} A^{-1} t$, меньших исходной плотности электронных ловушек (см. например, рис. $6, a)$.

Действительно, согласно [6,7] начальную кинетику зарядки можно оценить из соотношения

$$
\Delta Q_{\mathrm{tr}}=I_{0} t\left[\frac{E_{0}}{E_{i}} \delta_{0} \lambda_{\mathrm{eff}} n_{\mathrm{tr}}^{h} S_{\mathrm{tr}}^{h}-\left(1-\eta_{0}\right) R_{0} n_{\mathrm{tr}}^{e} S_{\mathrm{tr}}^{e}\right]
$$

где первый член в скобках отвечает за положительную зарядку, вызванную эмиссией ВЭ из слоя толщиной $\lambda_{\mathrm{eff}}$ с коэффициентом $\delta_{0}$ с созданием концентрации дырок $n_{\mathrm{tr}}^{h}$ при сечении захвата $S_{\mathrm{tr}}^{h}$, а второй член ответственен за отрицательную зарядку слоя толщиной $R_{0}$ с концентрацией электронных ловушек $n_{\mathrm{tr}}^{e}$ и сечением захвата $S_{\mathrm{tr}}^{e}$. Но оценить по формуле (20) величину аккумулируемого заряда и тем самым значения $V_{\mathrm{S}}(t)$ и $V_{\mathrm{S} 0}$ затруднительно, так как в литературе наблюдается большой разброс значений всех параметров, входящих в это соотношение. Времена зарядки до равновесного значения заряда и потенциала $V_{\mathrm{S} 0}$, определяемые оценочно по соотношениям (20) и (13), также различаются на порядки величин от экспериментальных. 

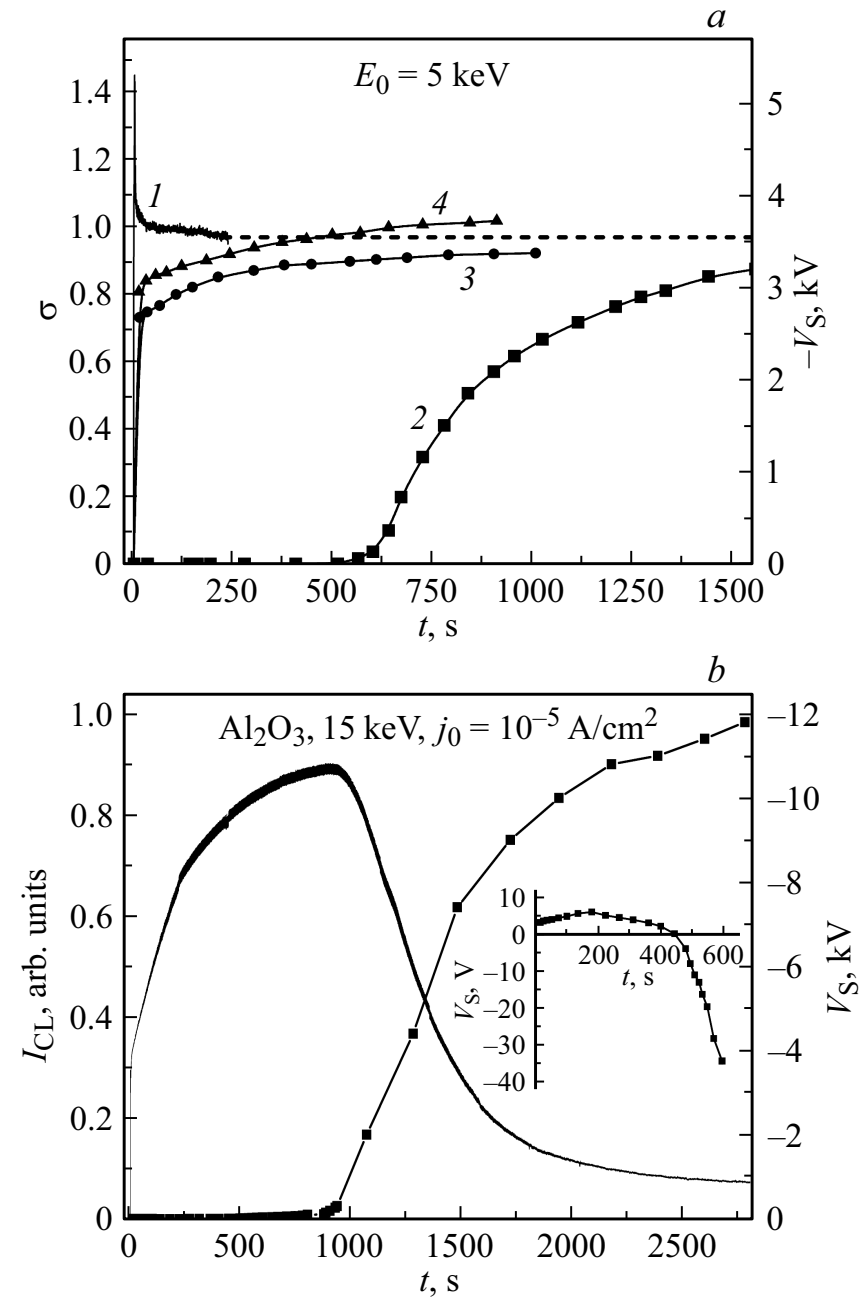

Рис. 7. (a) Временнб́е характеристики параметров зарядки сапфира при $E_{0}=5 \mathrm{keV} .1$ - зависимость $\sigma(t)$ для исходного сапфира, 2 - кинетика поверхностного потенциала $V_{\mathrm{S}}(t)$ для исходного сапфира, кривые 3 и 4 - кинетика поверхностного потенциала $V_{\mathrm{S}}(t)$ сапфира, предварительно облученного соответственно ионами $\left(10^{17} \mathrm{~cm}^{-2}\right)$ и электронами $\left(10^{20} \mathrm{~cm}^{-2}\right)$. (b) Временна́я зависимость потенциала поверхности $V_{\mathrm{S}}(t)$ и интенсивности катодолюминесценции исходного сапфира при $E_{0}=15 \mathrm{keV}$ и $j_{0}=10^{-5} \mathrm{~A} / \mathrm{cm}^{2}$. На вставке показана положительная зарядка сапфира на начальном этапе облучения.

Радиационные дефекты при электронном облучении возникают не только в органических диэлектриках (полимерах), но и в оксидах кремния $\mathrm{SiO}_{2}$ и алюминия $\mathrm{Al}_{2} \mathrm{O}_{3}$, а также других диэлектриках, что является, по мнению авторов работы [17], определяющей причиной зарядки $\mathrm{SiO}_{2}$, так как большинство ловушечных центров рождаются именно при электронной бомбардировке диэлектриков. Более того, в работе [35] утверждалось, что в некоторых „чистых“ монокристаллах, в частности $\mathrm{Al}_{2} \mathrm{O}_{3}$ (сапфир), зарядка вообще невозможна ввиду почти полного отсутствия изначальных ловушечных центров. Но эксперименты $[18,29,30]$ показали, что после набора некоторого критического значения дозы облучения сапфир также заряжается, что подтверждают измерения кинетики зарядки сапфира пучком электронов с плотностью тока $j_{0}=10^{-5} \mathrm{~A} / \mathrm{cm}^{2}$ при энергиях 5 и $15 \mathrm{keV}$, представленные на рис. 7. Эти результаты примечательны во многих отношениях. Во-первых, вопреки данным некоторых публикаций сапфир заряжается отрицательно при больших дозах облучения не только при $E_{0}=15 \mathrm{keV}$, но и уже при $E_{0}=5 \mathrm{keV}$, хотя кроссоверная энергия для сапфира $E_{20}=9 \mathrm{keV}$ по данным работы [8], и даже $E_{20}=15 \mathrm{keV}$ по данным работы [21]. Во-вторых, времена зарядки сапфира очень большие, на порядки величины больше, чем утверждалось в работах $[11,12]$, хотя времена достижения квазиравновесного значения коэффициента эмиссии $\sigma \approx 1$ намного меньше

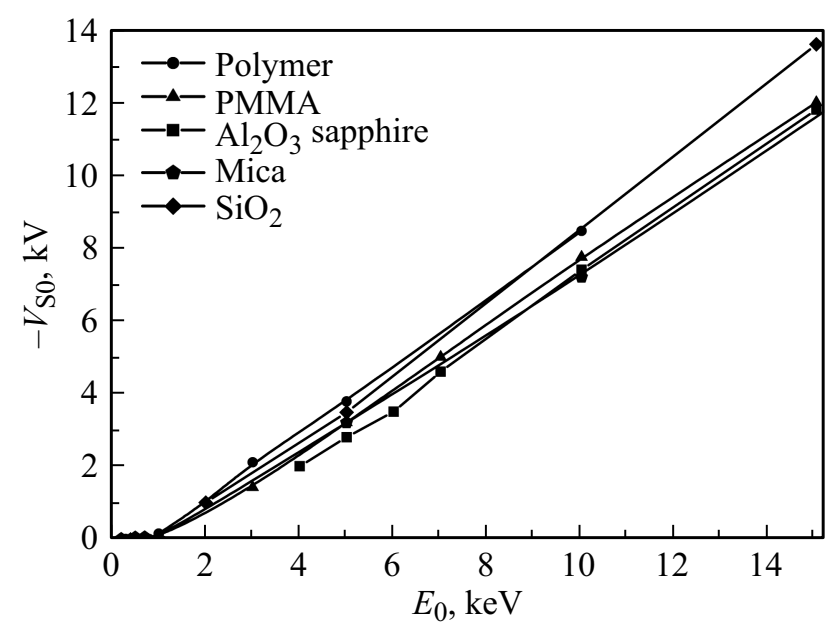

Рис. 8. Зависимость равновесного значения поверхностного потенциала $V_{\mathrm{S} 0}$ от энергии первичных электронов $E_{0}$ для различных образцов: полимера, ПММА, $\mathrm{Al}_{2} \mathrm{O}_{3}$ сапфира, слюды и $\mathrm{SiO}_{2}$.

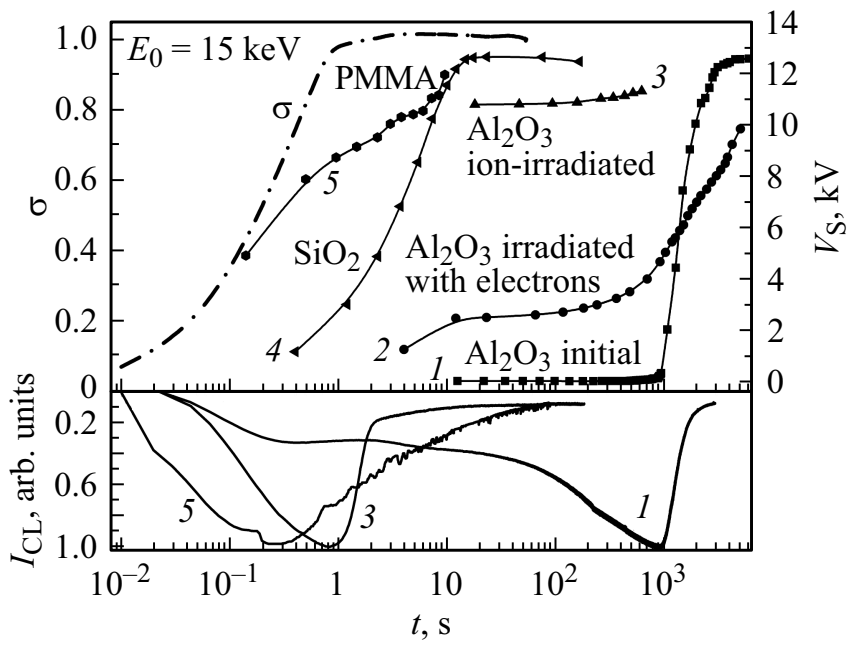

Рис. 9. Временна́я зависимость коэффициента эмиссии $\sigma(t)$, потенциала поверхности $V_{\mathrm{S}}(t)$ и интенсивности катодолюминесцентного излучения $I_{\mathrm{CL}}$, снятые при $E_{0}=15 \mathrm{keV}$ для различных образцов: исходный сапфир (кривая 1), сапфир, предварительно облученный электронами (кривая 2) и ионами (кривая 3), $\mathrm{SiO}_{2}$ (кривая 4) и ПММА (кривая 5). 
времени наступления равновесного потенциала зарядки поверхности. Примечательно, что если сапфир предварительно облучить определенной дозой электронов или ионов (рис. 7, $a$ ), то его зарядка происходит значительно быстрее и уже с начального момента облучения [29,30].

Все это указывает на тот факт, что вследствие очень малой концентрации изначальных собственных дефектов, а тем самым и ловушечных центров для электронов, сапфир долгое время не заряжается. Но в процессе облучения электронами или ионами в сапфире, как и в любом диэлектрике, возникают радиационно-стимулированные дефекты. После образования некоторой критической дозы облучения эти дефекты становятся центрами захвата электронов, вследствие чего происходит зарядка образца. Аналогичные закономерности были нами установлены и для монокристаллов кварца $\left(\mathrm{SiO}_{2}\right)$.

В заключение этого параграфа приведем сводные усредненные графики зависимостей равновесных отрицательных потенциалов поверхности $-V_{\mathrm{S} 0}\left(E_{0}\right)$ в зависимости от энергии облучающих электронов $E_{0}$ для ряда различных диэлектриков (рис. 8) и их временные характеристики (рис. 9), полученные в наших экспериментах. Их интерпретация дается в следующем разделе статьи.

\section{4. Объяснение полученных экспериментальных результатов и обоснование новой модели процессов зарядки диэлектриков при электронном облучении}

Из изложенного выше возникают три основных кардинальных вопроса. Первый - почему уже в начальные моменты облучения коэффициент эмиссии электронов $\sigma$ стремиться к единице, в то время как потенциал заряжающейся поверхности $-V_{\mathrm{S}}$ равен значению, далекому от равновесного значения в кроссоверной точке $E_{2 \mathrm{C}}$ ? Здесь налицо несостоятельность прежнего утверждения, что $\sigma \rightarrow 1$ вследствие уменьшения фактической энергии облучения $E_{L}=\left(E_{0}-q V_{\mathrm{S}}\right)$ до значения $E_{20}$. Практически зависимость $-q V_{\mathrm{S}}(t)$ в начале облучения изменяется намного медленнее, чем эмиссионная характеристика электронов $\sigma(t)$.

Второй вопрос - почему для некоторых диэлектриков (наиболее яркий пример - сапфир) при быстром достижении квазиравновесного значения $\sigma \approx 1$ образец еще долгое время почти не заряжается, а заметная зарядка начинается только при наборе определенной дозы облучения $D_{\mathrm{cr}}$ ? И третий вопрос - что является причиной столь близких значений равновесного потенциала зарядки поверхности различных диэлектриков?

Для объяснения этих ключевых факторов необходимо пересмотреть ряд принятых ранее допущений и моделей, так как в них не согласуются многие расчетные результаты с экспериментальными данными. Поэтому в настоящей новой модели мы вводим три новых положения, существенно меняющие общую картину механизма

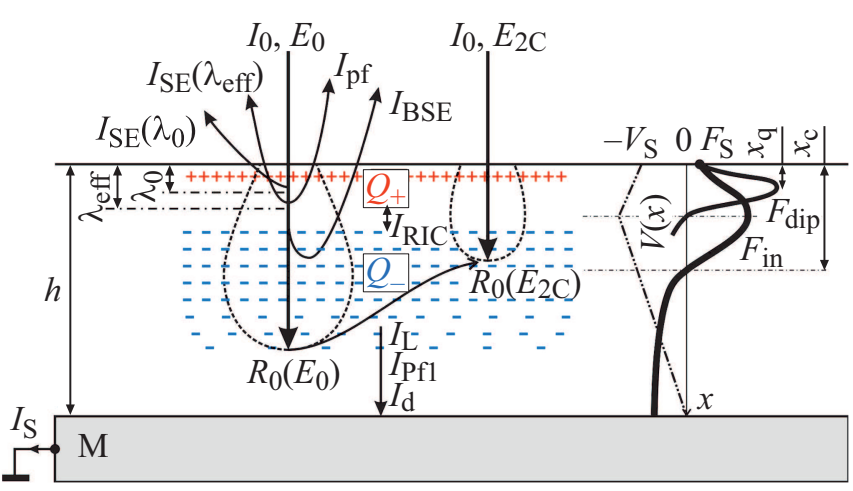

Рис. 10. Иллюстрация зарядки диэлектриков при электронном облучении и качественные зависимости потенциала зарядки $V(x)$, внутреннего поля $F_{\text {in }}$ и поля $F_{\text {dip }}$ дипольного слоя зарядов. $x_{q}$ - глубина смены знаков аккумулируемых зарядов, $x_{c}$ - глубина смены знаков внутреннего поля $F_{\text {in }}$.

зарядки диэлектрических мишеней. Первое - при достаточно высоких плотностях облучающих электронов, превосходящих плотности исходных ловушечных центров, часть первичных электронов, не захваченных на ловушки, после их термализации становится дополнительными свободными электронами с малыми энергиями (доли и единицы $\mathrm{eV}$ ). Эти первичные электроны в сильной степени подвержены влиянию образующемуся при зарядке внутреннему электрическому полю $F_{\text {in }}$ и под его действием движутся либо к поверхности, давая вклад в электронную эмиссию, либо движутся в необлученную область, давая вклад в радиационно-стимулированную проводимость, регистрируемую как ток утечки.

Второе ключевое положение - при электронной бомбардировке диэлектриков в них всегда образуются подпороговые радиационные дефекты, которые суммируются с исходными дефектами материала мишени. При малой плотности исходных ловушек именно радиационно-стимулированные дефекты являются основной причиной аккумуляции электронов и тем самым зарядки диэлектриков.

И, наконец, третье - регулирующим фактором установления равновесного состояния зарядки является образование критического поля двойного слоя зарядов $F_{\text {dip. }}$.

Учитывая эти три замечания, рассмотрим картину зарядки диэлектриков с привлечением наглядной иллюстрации на рис. 10. При энергии первичных электронов $E_{0}$ первоначальная глубина их проникновения равна $R_{0}\left(E_{0}\right)$, что примем за толщину формирующегося отрицательного слоя зарядов $Q_{-}$с центром тяжести в плоскости на расстоянии приблизительно $R_{0} / 3$ от поверхности. Положительный слой заряда $Q_{+}$формируется в слое, равном глубине выхода ВЭ $\lambda_{0} \ll R_{0}$. В настоящей работе примем для оценки глубины пробега электронов соотношение из работ $[11,12]: R_{0}[\mathrm{~nm}] \approx 93.4 E_{0}^{1.45} \rho^{-0.91}$, где $E_{0}$ выражено в $\mathrm{keV}, \rho-\left[\mathrm{g} / \mathrm{cm}^{3}\right]$. Между противоположно заряженными слоями $Q_{+}$и $Q_{-}$возникает сильное внутреннее поле $F_{\text {dip }}$, определяемое разностью 
потенциалов между этими слоями. В более общем случае, ввиду того что по абсолютной величине положительный потенциал $+V_{\mathrm{S}}$ много меньше отрицательного $-V_{\mathrm{S}}$, напряженность поля определяется как разность потенциалов между плоскостью центра тяжести отрицательного потенциала $\left(\approx R_{0} / 3\right)$ и расстоянием до ближайших, как правило заземленных, металлических элементов камеры микроскопа, например проводящей подложки, обозначенной $M$ на рис. 10 и равной толщине образца $h$, или входной плоскостью спектрометра над поверхностью образца на расстоянии $L$ (на рис. 10 не показана). На вакуумной поверхностной стороне образца поле $F_{\mathrm{S}}$ в зависимости от результирующего потенциала $V_{\mathrm{S}}(t)$ равно $F_{\mathrm{S}}(t)=-V_{\mathrm{S}}(t) / L$, в необлучаемом объеме $F(t)=-V_{\mathrm{S}}(t) /\left(h \varepsilon_{r}\right)$, а в подповерхностной области максимальное поле:

$$
F_{\text {in }}(t)=\frac{2 \Delta V_{\mathrm{S}}(t)}{R(t)-\lambda_{\mathrm{eff}}}=\frac{\sigma_{-}(t)}{\varepsilon_{0} \varepsilon_{r}}=\frac{\rho_{-}(t) R(t)}{\varepsilon_{0} \varepsilon_{r}},
$$

где $\Delta V_{\mathrm{S}}(t)$ есть разность абсолютных потенциалов между эквипотенциальными поверхностями на глубинах $R(t) / 3$ и $\lambda_{\text {eff }} / 2$ соответственно. Одновременно и независимо от поля $F_{\text {in }}$ формируется поле дипольного слоя зарядов $F_{\text {dip }}$ между положительным слоем $Q_{+}$и верхним слоем $Q_{-}$. Максимум этого поля находится на глубине $x_{q}$, в то время как максимум поля $F_{\text {in }}$ находится на большей глубине $x_{c}$ центроида отрицательного слоя зарядов $Q_{-}$. Именно под действием поля $F_{\text {dip }}$ возникает радиационностимулированный ток $I_{\mathrm{RIC}}$, который при достижении определенного критического значения $F_{\operatorname{dip}}^{\mathrm{cr}}$ стабилизирует процесс зарядки, приводя его к равновесному состоянию. В этом состоянии выполняется важное равенство $\frac{d Q_{\mathrm{tr}}}{d t}=I_{d}-I_{\mathrm{RIC}}=0\left(\right.$ при $\left.I_{L}=0\right)$, т. е. согласно $(11)$

$$
I_{0}\left(1-\eta_{0}\right) R_{2 \mathrm{C}} N_{\mathrm{tr}} S_{\mathrm{tr}} \approx \gamma \frac{\Delta V a^{2}}{R_{2 \mathrm{C}}} \approx \frac{I_{0} E_{L}}{E_{i}} \frac{\mu \tau \Delta V}{\left(R_{2 \mathrm{C}}-\lambda_{\mathrm{eff}}\right)^{2}},
$$

где $\mu$ и $\tau$ - это подвижность и время жизни свободных носителей заряда в облучаемой области. К сожалению, значение $\mu, \tau$ и $\Delta V$ не всегда доступны для случая исследования реальной поверхности диэлектрика. Поэтому величину $F_{\text {dip }}$ можно сейчас оценить только качественно.

Это равновесное состояние наступает при фактической энергии первичных электронов $E_{2 \mathrm{C}}=E_{0}-q V_{\mathrm{S} 0}$, которая много меньше $E_{0}$. Поэтому и глубина пробегов в равновесном состоянии $R_{0}\left(E_{2 \mathrm{C}}\right)$ меньше начального пробега $R_{0}\left(E_{0}\right)$, а координата центра тяжести отрицательного слоя смещается вверх, усиливая поле $F_{\text {in }}$ вследствие увеличения общего отрицательного заряда $Q_{-}$и одновременного уменьшения расстояния между двумя слоями $\left(R_{0}\left(E_{2 \mathrm{C}}\right) / 3-\lambda_{\mathrm{eff}} / 2\right)$. Здесь уместно подчеркнуть, что поле $F_{\text {in }}$ имеет максимум между слоями (см. рис. 10$)$, далее оно переходит через нулевое значение в центре отрицательной области, после чего изменяет свой знак на противоположный в необлучаемой части диэлектрика. Более точное выражение для поля $F_{\text {in }}$ приводится в работе [36], несколько измененной формой которого, мы будем пользоваться для оценки этого важнейшего параметра отрицательной зарядки

$$
F_{\text {in }}=\frac{\sigma_{-}}{\varepsilon_{0} \varepsilon_{r}}\left[1+\frac{h-0.3 R}{\varepsilon_{r} L}\right] .
$$

При $(h-0.3 R) / \varepsilon_{r} L \ll 1$ эта формула приводит к выражению (21) для тонкого заряженного слоя в свободном пространстве.

Эффект внутреннего поля $F_{\text {in }}$ сказывается также на частичном увеличении коэффициента выхода ВЭ, определяемого соотношением для $\delta_{\text {eff }}$ в формуле $(1)$. В этой формуле вместо значения $\lambda_{0}$ следует применять значение $\lambda_{\text {eff }}-$ среднюю глубину выхода ВЭ, которая увеличивается в поле $F_{\text {in }}$ по закону [11]:

$$
\lambda_{\text {eff }}=\lambda_{0} \exp \left( \pm \beta F_{\text {in }}\right)
$$

где $\beta$ - коэффициент затухания, $\beta \approx 1\left[\mathrm{~cm} \cdot \mathrm{MV}^{-1}\right]$. Например для $\mathrm{Al}_{2} \mathrm{O}_{3}$ в начальные моменты облучения при $E_{0}=15 \mathrm{keV} F_{\text {in }}=\frac{\Delta V_{\mathrm{S}}}{R_{0}}=\frac{10 \mathrm{~V}}{0.8 \mu \mathrm{m}}=1.2 \cdot 10^{5} \mathrm{~V} / \mathrm{cm}$, а в равновесии $F_{\text {in }} \approx 10^{6} \mathrm{~V} / \mathrm{cm}$. Расчет по формулам (1) и (24) дает увеличение коэффициента $\delta_{\text {eff }} / \delta_{0}$ в $1.3-1.5$ раза. Отсюда следует, что рост средней глубины выхода ВЭ $\lambda_{\text {eff }}$ при наличии поля $F_{\text {in }}$ вносит существенный вклад в увеличение коэффициента $\delta_{\text {eff. }}$ Такие дополнительные сопутствующие явления при наличии поля $F_{\text {in }}$, как уменьшение поверхностного барьера, уменьшение критического угла выхода ВЭ (относительно нормали к поверхности) [6,7] и освобождение электронов из мелких ловушек $[11,12]$, дают меньший эффект. Таким образом, рассмотренное влияние $F_{\text {in }}$ недостаточно для роста $\delta_{\text {eff }}$ и достижения квазиравновесного значения $\sigma \approx 1$ уже в начальные моменты облучения (см. рис. 5). Налицо существует еще одна веская причина увеличения числа эмиттированных электронов. Этот весьма примечательный факт впервые был обнаружен в работах [37,38], где на примере ряда диэлектриков показано, что при слабых дозах облучения $\left(j_{0} \leq 10^{-13} \mathrm{~A} / \mathrm{cm}^{2}\right.$ в секунду) кривая $\sigma=f\left(E_{0}\right)$ имеет минимум в области энергий $E_{0}$ вблизи $1 \mathrm{keV}$, а затем в области $E_{0}=1-10 \mathrm{keV}$ наблюдается значительный рост $\sigma$ вплоть до единицы. Авторы объясняют этот эффект возрастанием выхода низкоэнергетических отраженных электронов (с начальной энергией $E>50 \mathrm{eV}$ ) из-за возникающей отрицательной зарядки после длительного облучения. Возможно, это вопрос терминологии, но поле $F_{\text {in }}$ способно ускорить отраженные электроны при их баллистическом движении к поверхности только на единицы $\mathrm{eV}$, что составляет относительно малую величину для всех OЭ с энергией $E>50 \mathrm{eV}$.

Помочь ответу на вопрос, откуда дополнительно берется значительное число эмиттированных электронов в рассмотренной ситуации, может следующее рассуждение. Только малая часть первичных электронов рассеивается обратно (коэффициент ОЭ $\eta_{0} \approx 0.1$ ), в то время как их основная доля $\left(1-\eta_{0}=\alpha\right)$ после 
потерь энергии в процессах неупругих столкновений в конце их траекторных пробегов термализуются, и их остаточная энергия составляет доли и единицы $\mathrm{eV}$. Для этих „медленных“ электронов, локализованных во всей области взаимодействия первичных электронов с мишенью, даже слабое начальное внутреннее поле $F_{\text {in }}$ (порядка $10^{4} \mathrm{~V} / \mathrm{cm}$ ) вызывает их направленное движение к поверхности и в сторону подложки. Если энергия этих ускоренных в поле $F_{\text {in }}$ электронов больше электронного сродства диэлектрика $(\chi \approx 1)$, то они преодолеют потенциальный барьер и эмиттируются с энергией, сравнимой с низкоэнергетическими вторичными электронами. Ток эмиссии этих электронов в вакуум обозначим через $I_{\mathrm{pf}}$ (см. рис. 10), а часть термализованных первичных электронов, идущих на подложку, через $I_{\mathrm{pf} L}$. Отметим, что эти эмиттированные электроны в некотором роде аналогичны генерируемым в известном эффекте Малтера, где также наблюдается кратное увеличение эмиссии ВЭ под воздействием внутреннего электрического поля. Но есть и существенные различия. Эффект Малтера присущ тонким диэлектрическим слоям на проводящей подложке, являющейся, по сути, резервуаром свободных электронов. В нашем случае диэлектрическая мишень „толстая“, практически не проводящая, поэтому приток свободных электронов со стороны подложки исключен. По этой причине считаем, что первичные термализованные электроны как раз и являются тем источником, который уже в начальные моменты облучения обеспечивают быстрый рост коэффициента $\sigma$ до единицы, что было наглядно продемонстрировано на всех представленных выше рисунках.

Увеличение $\sigma$ за счет вводимого нами члена с коэффициентом $\alpha=I_{\mathrm{pf}} / I_{0}$ требует корректировки выражения (15) для баланса токов и зарядов

$$
I_{0}=I_{0}\left(\sigma_{\mathrm{SEB}}+\alpha\right)+\frac{d Q_{\mathrm{tr}}}{d t}+\left(I_{L}+I_{\mathrm{pf} L}\right),
$$

где первый член выражает ток эмиссии электронов, включающий ВЭ, ОЭ и новую составляющую $I_{0} \alpha$ термализованных первичных электронов, а к току утечки добавляется вклад части термализованных электронов $I_{\mathrm{pf} L}$. Естественно, что указанное перераспределение токов справедливо только в том случае, когда число входящих электронов $N_{0}$ больше числа изначальных ловушечных центров $N_{\text {in }}$ для электронов в облучаемом объеме образца.

Отметим теперь тот существенный факт, что указанные термализованные первичные электроны не вносят никакого влияния на электризацию диэлектрика, так как они не захватываются на ловушки, т.е. не аккумулируются в материале мишени, но в то же время они увеличивают токи эмиссии и утечки. Поэтому следует быть крайне осторожным при расчете токов эмиссии, смещения и утечки и при сравнении этих расчетных данных с экспериментальными измерениями, так как пока не вполне ясно как вычленить и оценить величину вклада термализованных первичных электронов в токи эмиссии, утечки и смещения.

Если выполняется условие $N_{0} \approx N_{\text {in }}$, то большинство рассмотренных выше моделей зарядки при учете токов $I_{\mathrm{pf}}$ справедливы (включая быстрые времена зарядки), но реально при выбранных параметрах эксперимента значение $N_{\text {in }}$ оказывается слишком малым, чтобы завершить процесс зарядки в начальные короткие времена облучения $t_{1}$. После времени $t_{1}$ зарядка продолжается, но теперь уже за счет перманентного образования новых радиационных дефектов $N_{\text {rad }}$, стимулирующих дополнительный захват на них первичных электронов. Этот момент очень важен в нашей модели зарядки, однако он умозрительно предполагался лишь в единичных публикациях $[19,20,39,40]$. Известно, что сечение подпорогового радиационного дефектообразования на порядки величин меньше сечения захвата носителей на ловушки. Более того, захват носителей начинается только после достижения определенной критической дозы облучения $[39,40,41]$. К сожалению вопрос о радиационном подпороговом дефектообразовании и величине критической дозы в различных диэлектриках еще мало изучен, поэтому наши дальнейшие численные оценки носят лишь приближенный характер.

Итак, с самого первого момента облучения мишени электронами с плотностью $j_{0}$ (за время $t_{1}$ в интервале до десятых долей и единиц секунд) быстро заполняются исходные мелкие ловушечные центры с концентрацией $N_{\text {in. }}$ Если при этом не достигается критическое значение поля зарядов $F_{\text {in }}^{\mathrm{C}}$, то зарядка продолжается за счет глубоких ловушек $n_{\text {rad }}\left(t_{2}\right)$, порождаемых за более длительное время $t_{2}$ вплоть до наступления равновесного состояния, контролируемого полем $F_{\text {in }}^{\mathrm{C}}$. Заметим, что на определяющую роль $F_{\text {in }}$ при зарядке диэлектриков указывалось также в работах $[42,43]$.

В итоге новое уравнение баланса зарядов, учитывающее процессы заполнения и опустошения как мелких, так и глубоких ловушечных центров, для электронов можно с учетом члена $n_{\text {rad }}\left(t_{2}\right)$ представить следующим уравнением (вместо уравнения (18)):

$$
\begin{aligned}
& \frac{d n_{\mathrm{tr}}\left(t_{1}, t_{2}\right)}{d t}=\frac{\left(1-\eta_{0}-\alpha_{0}\right) j_{0} S_{\mathrm{tr} 1,2}}{q}\left[\left[N_{\mathrm{in}}+n_{\mathrm{rad}}\left(t_{2}\right)\right]\right. \\
& \left.-n_{\mathrm{tr}}\left(t_{1}, t_{2}\right)\right]-n_{\mathrm{tr}}\left(t_{1}, t_{2}\right) f_{1} \exp \left[-\frac{E_{T}-\Delta E_{\mathrm{PF}}\left(t_{2}\right)}{k T}\right],
\end{aligned}
$$

где $\Delta E_{\mathrm{PF}}\left(t_{2}\right)=\beta F_{\text {in }}^{1 / 2}\left(t_{2}\right)$ определяется из выражения (19), а $S_{\mathrm{tr} 1}$ - сечение захвата мелких ловушек c энергией $E_{\mathrm{T} 1}, n_{\mathrm{tr}}\left(t_{1}\right)$ - число текущего заполнения изначальных ловушек в единице объема; $n_{\text {rad }}\left(t_{2}\right)-$ число генерируемых при облучении радиационных подпороговых дефектных центров прилипания, $f_{1}-$ скорость освобождения электронов из ловушечных центров с энергией $E_{\mathrm{T}}$ из-за понижения потенциального барьера $\Delta E_{\mathrm{PF}}\left(t_{1}\right)$ под воздействием поля $F_{\text {in }}(t)$. Учет члена $n_{\mathrm{rad}}\left(t_{2}\right)$ был впервые предложен в работе [44]. 
Ввиду большой разницы времен заполнения исходных ловушек ( $t_{1}$ порядка десятков $\left.\mathrm{ms}\right)$ с концентрацией $N_{\text {in }}$ и времени $t_{2}$ образования радиационно-стимулированных ловушек (от единиц до сотен секунд) с концентрацией $n_{\text {rad }}\left(t_{2}\right)$, целесообразно разделить уравнение (26) на две части, где первый член $\frac{d n_{\mathrm{tr} 1}\left(t_{1}\right)}{d t}$ будет ответственен за быстрое заполнение исходных мелких ловушек, а второй за рождение и заполнение глубоких ловушек $n_{\text {rad }}\left(t_{2}\right)$. Предварительно обозначим константы в (26) следующим образом:

$$
A=N_{\text {in }}, \quad B=f_{1} \exp \left[-\frac{E_{T}-\beta F_{\text {in }}^{1 / 2}}{k T}\right], \quad C=\frac{j_{0} S_{\mathrm{tr} 1}}{q} .
$$

Тогда первый член уравнения (26) запишется в виде (при допущении $\left.E_{T}=\beta F_{\text {in }}^{1 / 2)}\right)$ :

$$
\frac{d n_{\mathrm{tr} 1}\left(t_{1}\right)}{d t_{1}}=C A-(C+B) n_{\mathrm{tr} 1}
$$

а его решение при интегрировании от $t_{1}=0$ до $t_{1}=t_{1}^{0}$

$$
n_{\mathrm{tr} 1}=\frac{C A-\left[C A-(C+B) N_{01}\right] \exp \left\{-(C+B)\left(t_{1}-t_{1}^{0}\right)\right\}}{C+B} .
$$

Аналогичным путем находится $\frac{d n_{\text {rad }}\left(t_{2}\right)}{d t_{2}}$ для глубоких ловушек с энергией $E_{\mathrm{T} 2}$ и сечением захвата $S_{\mathrm{tr} 2}$ :

$$
\begin{aligned}
\frac{d n_{\mathrm{tr}}\left(t_{2}\right)}{d t_{2}}= & \frac{\left(1-\eta_{0}-\alpha_{0}\right) j_{0} S_{\mathrm{tr} 2}}{q}\left[N_{0}^{s}+\int_{0}^{t_{2}} G_{\mathrm{rad}} d t_{2}\right] \\
& -n_{\mathrm{rad}}\left(t_{2}\right) f_{2} \exp \left[-\frac{E_{\mathrm{T} 2}-\Delta E_{\mathrm{PF}}}{k T}\right],
\end{aligned}
$$

где $N_{0}^{S}$ - число заполненных ловушек в равновесном состоянии.

К сожалению, из-за трудности определения скорости генерации радиационных центров $G_{\text {rad }}(t)$, их энергии активации и сечения захвата, представленные уравнения поддаются только качественному анализу.

Дальнейшее изложение целесообразно сопровождать численными оценками, например для сапфира $\left(\mathrm{Al}_{2} \mathrm{O}_{3}\right)$. Ключевым параметром является значение внутреннего поля $F_{\text {in }}$, регулирующее темпы заполнения (захвата электронов) и опустошения ловушечных центров с энергией активации от 0.1 до $1 \mathrm{eV}$ (мелкие ловушки) и величина равновесного потенциала $V_{\mathrm{S} 0}$. Так как величина $F_{\text {in }}$ заранее неизвестна, то определим ее по экспериментально измеренному равновесному значению поверхностного потенциала. Например $V_{\mathrm{S} 0}=10 \mathrm{kV}$ при $I_{0}=10^{-9} \mathrm{~A}$, $a=100 \mu \mathrm{m}, E_{0}=13 \mathrm{keV}, \chi=E_{\mathrm{T}}=1 \mathrm{eV}, d=1 \mathrm{~mm}$. To есть

$$
V_{\mathrm{S} 0}=\frac{q N_{1 \mathrm{tr}}^{S} d}{\varepsilon_{0} \varepsilon_{r}}=\frac{Q d}{\varepsilon_{0} \varepsilon_{r} a^{2}}=10^{4} \mathrm{~V}
$$

из чего следует, что максимальная равновесная поверхностная плотность зарядов $N_{1 \mathrm{tr}}^{S}=10^{-7} \mathrm{C} \cdot \mathrm{cm}^{-2}$ или $10^{12}$

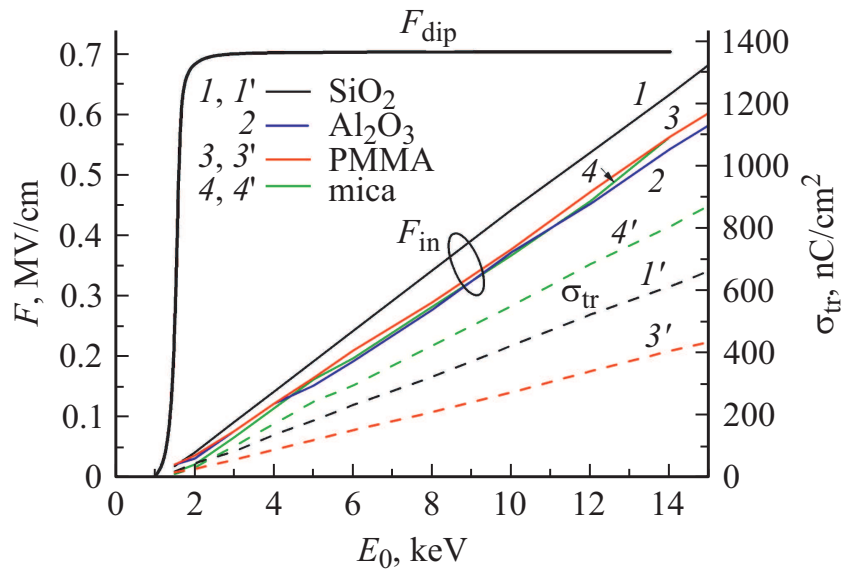

Рис. 11. Графики зависимостей электрических полей $F_{\text {in }}$ и $F_{\text {dip }}$ от энергии $E_{0}$ для ряда диэлектриков, а также характеристики плотности аккумулированных отрицательных зарядов $\sigma_{\mathrm{tr}}=f\left(E_{0}\right)$ (штриховые кривые).

ловушек в $\mathrm{cm}^{-2}$, т.е. $N_{1 \mathrm{tr}}=10^{16} \mathrm{~cm}^{-3}$. Отсюда критическое поле равно $F_{\text {in }}=\frac{N_{1 \mathrm{tr}}^{S}}{\varepsilon_{0} \varepsilon_{r}}=10^{5} \mathrm{~V} / \mathrm{cm}$, а равновесное значение зарядов $Q_{\mathrm{tr}}=10^{-9} \mathrm{C}$ набирается за сотни секунд, т.е. на зарядку идут десятые или сотые доли от входящих электронов, а основная их часть уходит в потоки эмиттированных термализованных электронов и в токи утечки.

Для различных классов диэлектриков это поле приблизительно постоянно, так как $V_{S}\left(E_{0}\right) \approx$ const для всех диэлектриков при заданном $E_{0}$ (см. рис. 8), поэтому $\approx Q_{\text {tr }}$ отличается для них в основном из-за различия в параметре $\varepsilon_{r}$, что подтверждается экспериментально.

Подтверждением близости значений $F_{\text {in }}$ для всех диэлектриков являются представленные на рис. 11 характеристики $F_{\text {in }}$ как функции энергии облучающих электронов $E_{0}$ для ряда диэлектриков, а также универсальная зависимость $F_{\text {dip }} \approx$ const для всех образцов. Бросается в глаза, что $F_{\text {in }}$ близки по значению для всех диэлектриков, несмотря на существенные различия в фундаментальных параметрах $\varepsilon_{r}, \rho_{m}, \beta$. В отличие от схожести в характеристиках полей $F_{\text {in }}$ и $F_{\text {dip }}$, поверхностные плотности зарядов $\sigma_{\text {tr }}$ сильно различаются для различных диэлектриков, что показано на рис. 11 штриховыми кривыми. Объяснение этому, довольно простое, вытекает из соотношения, связывающего поле $F_{\text {in }}$ с плотностью зарядов $\sigma_{\mathrm{tr}}$ и диэлектрическими константами $\varepsilon_{r}$ : $F_{\text {in }}=\frac{\sigma_{\text {tr }}}{2 \varepsilon_{0}\left(\varepsilon_{r}+1\right)}$. Что касается постоянства поля $F_{\text {dip }}$, то этот факт вытекает из близости значений $F_{\text {dip }}=\frac{\Delta V}{2 x_{q}}$ для всех диэлектриков. К сожалению значения $\Delta V$ и особенно $x_{q}$ трудно рассчитать, поэтому график $F_{\text {dip }}$ на рис. 11 следует считать не количественным, а качественным, дающим общее представление о необычной характеристике $F_{\text {dip. }}$ Более наглядно поведение генерируемых потенциалов и полей заряженных диэлектриков демонстрируется на показательном рис. 12. Координаты 


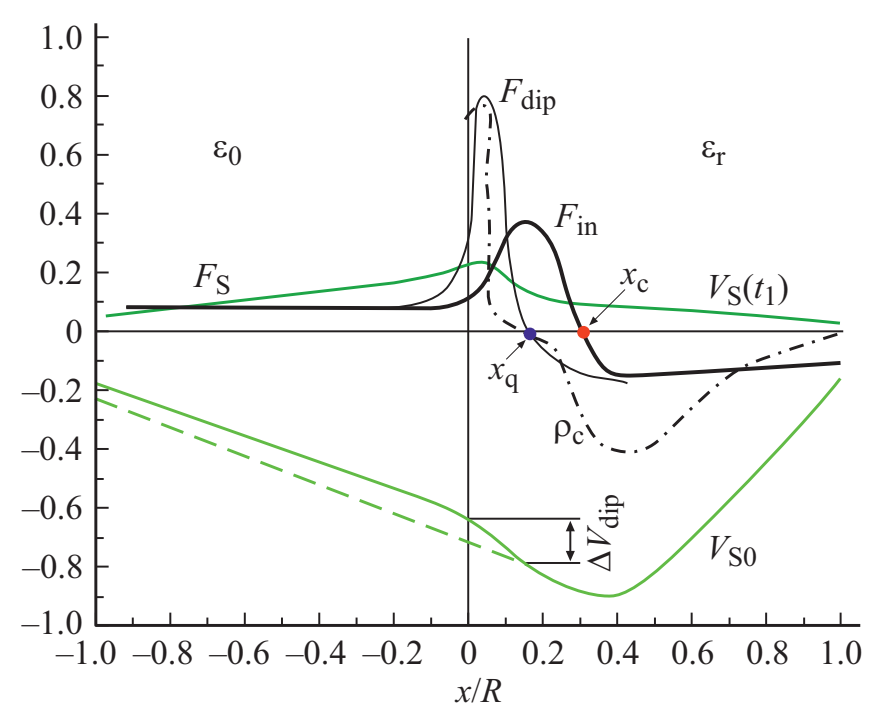

Рис. 12. Приведенные эмпирические характеристики распределения зарядов $\rho_{c}$ в состоянии равновесия (штриховая кривая), потенциалов $V_{\mathrm{S}}(t)$ в начальный момент зарядки и $V_{\mathrm{S} 0}$ в состоянии равновесия, а также электрических полей $F_{\text {in }}$ в облучаемом объеме диэлектрика и $F_{\text {dip }}$ в дипольном слое в равновесном состоянии зарядки. $x_{q}$ - глубина смены знака зарядов (порядка $15-50 \mathrm{~nm}), x_{c}$ - глубина центроида отрицательных зарядов, где происходит смена знака поля $F_{\text {in. }}$.

на рис. 12 даются в условных единицах, также как и кардинальные точки $x_{q}$ и $x_{c}$. Приведенные на этом рисунке качественные характеристики распределения зарядов и полей довольно хорошо описываются при следующей аппроксимации:

$$
\rho(x) / \rho_{0}=\left(0.5-B\left(x / x_{q}\right)^{2}\right) \exp \left[-B\left(x / x_{q}-1 / B\right)^{2}\right],
$$

где $B=\left(x_{q} / R_{0}\right)^{-1}, \rho_{0}-$ плотность зарядов на поверхности. Отсюда распределение поля в дипольном слое зарядов, согласно уравнению Пуассона, выражается формулой

$$
\begin{aligned}
\frac{E(x)}{E_{0}}= & \frac{1}{\varepsilon_{0}\left(\varepsilon_{r}+1\right)} \int \frac{\rho(x)}{\rho_{0}} d x \\
= & \frac{(B x+1) \exp \left[-(B x-1)^{2} / B\right]}{2 B} \\
& -\frac{\sqrt{\pi}[(2 A-1) B-2] \operatorname{erf}[(1-B x) / \sqrt{B}]}{4 B^{3 / 2}},
\end{aligned}
$$

где erf - функция ошибок, $A$ и $B-$ подгоночные параметры, зависящие от материала образца. Качественно результаты хорошо коррелируют с работами $[6,7,11,12]$.

Зная $F_{\mathrm{cr}}, N_{\mathrm{tr}}$ и $f$ можно по соотношению (28) найти расчетное ожидаемое время зарядки $t_{1} \approx 200 \mathrm{~ms}$, что, в общем-то, согласуется с данными других теоретических работ. Но учитывая парадоксальное различие этого расчетного значения с экспериментом, дающим, например, для $\mathrm{Al}_{2} \mathrm{O}_{3}$ значение $t_{1} \approx 500-1000 \mathrm{~s}$, приходим к выводу, что изначально нет концентрации исходных ловушек
$N_{1 \mathrm{tr}}=10^{16} \mathrm{~cm}^{-3}$, а их число на четыре порядка меньше, т. е. порядка $10^{12} \mathrm{~cm}^{-3}$. Таким образом за $0.2 \mathrm{~s}$ облучения потенциал зарядки $V_{\mathrm{S}}(t)$ на $\mathrm{Al}_{2} \mathrm{O}_{3}$ также достигает величины всего около $1 \mathrm{~V}$, что мы и видим из экспериментального значения на рис. 9. Чтобы достичь равновесной концентрации дефектных уровней $N_{1 \mathrm{tr}}^{\mathrm{C}}$ требуется облучать сапфир при выбранном потоке облучающих электронов в течение длительного времени $t_{2} \approx 1000 \mathrm{~s}$. За это время образуется $N_{\text {rad }}=10^{16} \mathrm{~cm}^{-3}$ радиационных ловушечных дефектов со скоростью $10^{13} \mathrm{~cm}^{-3}$ в секунду. Кинетика этого второго этапа зарядки согласуется с расчетом второго члена в фигурных скобках уравнения (26).

Аналогичные временные характеристики процесса зарядки многих диэлектрических мишеней подтверждают принятую модель с решающей ролью радиационностимулированного дефектообразования.

С учетом изложенных фактов зависимость поверхностного потенциала $V_{\mathrm{S}}$ от дозы облучения $j_{0} t$ можно представить также как сумму двух экспонент, отвечающих за зарядку исходно имеющихся центров захвата электронов $N_{\text {in tr }}$ и радиационно генерированных центров $N_{\text {rad. }}$. Так как при $N_{\text {tr }}^{\mathrm{C}} \approx$ const число $N_{\text {intr }}=N_{\text {tr }}^{\mathrm{C}}-N_{\text {rad }}$ в различных диэлектриках может отличаться на порядки, то и время равновесной зарядки различаются на порядки величин. Здесь необходимо сделать существенную ремарку. В значение времени $t_{2}$ входят две составляющие: $t_{2 \operatorname{tr}}$ и $t_{0}$, то есть $t_{2}=t_{0}+t_{2 \mathrm{tr}}$, где $t_{0}$ есть время критического допорогового дефектообразования, во время которого мишень практически не заряжается $\left(t_{0} \approx 500 \mathrm{~s}\right.$ на рис. 7,a). Это, возможно, объясняется тем, что сечение допорогового радиационного дефектообразования очень мало $\left(S_{\mathrm{rad}} \approx 10^{-20}-10^{-25} \mathrm{~cm}^{2}\right)$ [45] по сравнению с сечением захвата электронов $\left(S_{\mathrm{tr}} \approx 10^{-12}-10^{-13} \mathrm{~cm}^{2}\right)$ при сравнительно низких плотностях исходных дефектов $\left(N_{\text {in }}=10^{13}-10^{16} \mathrm{~cm}^{-3}\right)$. За время $t_{0}$ происходит генерация нестабильных возбужденных состояний ловушечных уровней, на которых с большой вероятностью рекомбинируют носители, сопровождаясь катодолюминесцентным (КЛ) излучением (рис. 7, b, рис. 9). Так для исследованного нами сапфира в течение сотен секунд интенсивность КЛ с длиной волны 0.4-0.45 $\mu \mathrm{m}$ возрастала, приходя в насыщение. Это означает, что плотность излучательных центров с энергией фотонов порядка $3 \mathrm{eV}$ вначале увеличивается, достигая максимума, а затем приходит в насыщение. Голубое свечение сапфира с длинной волны $0.4-0.45 \mu \mathrm{m}$ обусловлено рекомбинацией носителей на уровнях, имеющих энергию активации 3 или $3.8 \mathrm{eV}$, что присуще генерации и рекомбинации при наличии $\mathrm{F}$ и $\mathrm{F}^{+}$глубоких центров, например вакансий кислорода [18]. Но что характерно, такое же КЛ-свечение наблюдается у ряда полимеров, например у полиметилметакрилата (ПММА), что продемонстрировано на том же рис. 9. Спад КЛ интенсивности вызван возможным частичным незначительным гашением (затуханием), но в основном уменьшением энергии 
облучающих электронов $E_{\mathrm{L}}\left(t_{2}\right)=E_{0}-e V_{\mathrm{S}}\left(t_{2}\right)$ за время облучения от $t_{0}$ до $t_{2}$.

Из сопоставления графиков зависимости $I_{\mathrm{CL}}(t)$ и $V_{\mathrm{S}}(t)$ на рис. $7, b$ и рис. 9 следует очередное подтверждение, что зарядка диэлектриков происходит в два этапа на первом этапе аккумулируются электроны на исходных ловушках, в основном мелких, на втором - на глубоких, радиационно-генерируемых. Но примечательно, что радиационно-генерированные ловушки, способные аккумулировать электроны, возникают не сразу, а после накопления определенной критической дозы облучения. Как видно из графиков на рис. 9 этот процесс начинается после того, как темп генерации-рекомбинации неравновесных носителей становиться постоянным. Можно предположить, что, только начиная с этого периода облучения, начинают формироваться стабильные долгоживущие дефекты, способные захватывать электроны на энергетические уровни этих образующихся центров [40,41]. Таким образом, КЛ-излучение можно с успехом использовать для сравнительных кинетических исследований зарядки, как для радиационно-стойких, так и чувствительных к облучению диэлектриков.

К сожалению, в настоящее время нам не известны количественные теоретические модели, позволяющие полно и адекватно описать временную эволюцию допорогового дефектообразования в диэлектриках различного класса. Эксперименты лишь показывают, что времена $t_{0}$ предварительного накопления нестабильных состояний, то есть набор критической дозы $D_{\mathrm{cr}}$, очень различны - от долей и единиц секунд для полимерных диэлектриков (радиационно чувствительные материалы) до сотен и тысяч секунд для радиационно-стойких (такие как сапфир). Из представленных на рис. 9 характеристик $I_{\mathrm{CL}}(t)$ и $V_{\mathrm{S}}(t)$ следует, что времена зарядки $t_{2}$ либо равны по порядку величины времени $t_{0}$ набора дозы $D_{\text {cr }}$ (для $\mathrm{Al}_{2} \mathrm{O}_{3}$ ), либо $t_{0}<t_{1}, t_{2}$ как, например у ПММА.

На основе изложенного, для описания временной зависимости потенциала поверхности $V_{\mathrm{S}}(t)$ диэлектрика при облучении электронами средних энергий $(1-30 \mathrm{keV})$ приведем следующую эмпирическую формулу:

$$
\begin{aligned}
V_{\mathrm{S}}(t)= & \frac{Q_{\mathrm{in}}}{\varepsilon_{0} \varepsilon_{r} a}\left[1-\exp \left(-\frac{Q_{\mathrm{tr}}\left(t_{1}\right)}{Q_{\mathrm{in}}}\right)\right] \\
& +\frac{Q_{\mathrm{rad}}}{\varepsilon_{0} \varepsilon_{r} a}\left[1-\exp \left(-\frac{Q_{\mathrm{rad}}\left(t_{2}\right)}{Q_{\mathrm{sat}}}\right)\right],
\end{aligned}
$$

где $Q_{\text {in }}-$ заряд исходно существующих дефектных центров в объеме облучения, $Q_{\mathrm{rad}}$ - заряд радиационногенерируемых ловушечных центров, $Q_{\text {sat }}-$ равновесный заряд $Q_{\mathrm{sat}}=Q_{\mathrm{in}}+Q_{\mathrm{rad}}$, при котором потенциал $V_{\mathrm{S}}$ приходит в равновесное насыщение. Первый член выражения (29) отвечает за быструю, второй - за долговременную составляющую процесса зарядки. Если $Q_{\text {in }} \approx Q_{\text {sat }}$, то равновесный потенциал устанавливается довольно быстро для всех диэлектриков, если $Q_{\text {in }} \ll Q_{\text {sat }}$, то доминирует второй, долговременный член выражения (30).

\section{5. Заключение}

В работе проведена ревизия существующих разрозненных, и во многом противоречивых представлений о фундаментальных явлениях в процессе зарядки диэлектрических мишеней при электронном облучении и предложена практически новая концепция радиационной зарядки, более согласующаяся с экспериментальными данными. Усовершенствованная модель зарядки приводит к следующим выводам.

1. Решающую роль в установлении электростатического равновесного потенциала зарядки диэлектрических мишеней играет критическая доза облучения, которая отвечает за возникновение внутреннего критического поля, по порядку величины равного для всех классов диэлектриков и для всех энергий первичных облучающих электронов.

2. Значение равновесного потенциала $V_{\mathrm{S} 0}$, как правило, не равно значению $\left(E_{0}-E_{20}\right) / q$, где предполагалось равновесное значение коэффициента полной эмиссии $\sigma_{\text {eff }}=1$. Равновесная энергия облучающих электронов $E_{\mathrm{L}}$ может быть как больше, так и меньше значения $E_{20}$.

3. Для кинетики зарядки и временных характеристик большое значение имеют первичные термализованные электроны, то есть доля первичных электронов, которые потеряли свою энергию вплоть до тепловых значений, но не были захвачены ловушками. Эти „медленные“ первичные электроны выносятся внутренним полем $F_{\text {in }}$ за пределы области облучения и дают вклад как в ток эмиссии вторичных электронов, так и в ток радиационной проводимости на заземленную подложку, но не вносят вклад в процесс аккумуляции зарядов, то есть непосредственно в процесс зарядки мишени.

4. Определяющим фактором времени установления равновесной зарядки является значение предельной концентрации ловушек в диэлектрике, являющееся суммой исходно присутствующих ловушек и вновь генерированных в процессе радиационного допорогового дефектообразования. При этом мелкие ловушки играют существенной роль на первом (быстром) этапе зарядки, так как они приходят в равновесие в процессе „заполнение-опустошение“ из-за эффекта Poole-Frenkel. Глубокие ловушки образуются в процессе радиационного дефектообразования после набора определенной критической дозы, зависящей от степени радиационной стойкости материала образца. После получения образцом этой дозы глубокие уровни ловушек дефектных центров начинают невосполнимо и устойчиво аккумулировать электроны, заряжая мишень до критической плотности вновь созданных ловушек, адекватной предельному критическому значению генерируемого внутреннего поля.

Главной движущей силой саморегулирующегося самосогласующегося процесса зарядки диэлектриков при электронном облучении выступает не только коэффициент эмиссии электронов, как общепринято считалось ранее, а формирование электрического поля дипольного 
слоя зарядов. Это критическое регулирующее поле $F_{\mathrm{cr}}$ порядка $0.5 \mathrm{MV} / \mathrm{cm}$ приблизительно одинаково для всех диэлектриков при любых значениях $E_{0}$. Однако следует признать, что это утверждение требует дальнейшего детального теоретического анализа и экспериментальной проверки.

\section{Финансирование работы}

Работа выполнена при поддержке гранта РФФИ № 18-02-00813.

\section{Конфликт интересов}

Авторы заявляют, что у них отсутствует конфликт интересов.

\section{Список литературы}

[1] L.B. Schein. Science 316, 1573 (2007).

[2] H. Miyake, K. Nitta, S. Michizono, Y. Saito. J. Vac. Soc. Jpn 50, 378 (2007).

[3] М.И. Панасюк, Л.С. Новиков. Модель космоса. Изд-во КДУ, М. (2007).

[4] И.М. Бронштейн, Б.С. Фрайман. Вторичная электронная эмиссия. Наука, М. (1969).

[5] L. Reimer. Scanning Electron Microscopy: Physics of image formation and microanalysis. Springer, Berlin (1998).

[6] J. Cazaux. Nucl. Instr. Meth. Phys. Res. B 244, 307 (2006).

[7] J. Cazaux. J. Electr. Spectr. Rel. Phenomena 176, 58 (2010).

[8] A. Melchinger, S. Hofmann. J. Appl. Phys. 78, 6224 (1995).

[9] Е.Н. Евстафьева, Э.И. Рау, В.Н. Милеев, Л.С. Новиков, С.А. Дицман, Р.А. Сеннов. Перспективные материалы 4, 11, (2010).

[10] I.A. Glavatskikh, V.S. Kortov, H.-J. Fitting. J. Appl. Phys. 89, 1, 44 (2001).

[11] M. Touzin, D. Goeuriot, C. Guerret-Piecourt, D. Juve, D. Treheux, H.-J. Fitting. J. Appl. Phys. 99, 114110 (2006).

[12] N. Cornet, D. Goeuriot, C. Guerret-Piecourt, D. Juve, D. Treheux, M. Touzin, H.-J. Fitting. J. Appl. Phys. 103, 064110 (2008).

[13] V.V. Aristov, L.S. Kokhanchik, K.P. Meyer, H. Blumtriff. Phys. Status Solidi A 78, 229 (1983).

[14] L. Frank, M. Zadrazil, I. Mullerova. Microchim. Acta 13, 289 (1996).

[15] M. Belhaj, O. Jbara, M.N. Filippov, E.I. Rau, M.V. Andrianov. Appl. Surf. Sci. 177, 58 (2001).

[16] S. Fakhfakh, O. Jbara, M. Belhaj, Z. Fakhfakh, A. Kallel, E.I. Rau. Eur. Phys. J. Appl. Phys. 21, 137 (2003).

[17] H. Gong, C. Ong. J. Appl. Phys. 75, 449 (1994).

[18] J. Liebault, K. Zarbout, D. Moya-Siesse, J. Bernardini, G. Moya. Appl. Surf. Sci. 9852, 1 (2003).

[19] A. Boughariou, G. Blaise, D. Braga, A. Kallel. J. Appl. Phys. 95, 4117 (2004).

[20] K. Zarbout, A. Ahmed, G. Moya, J. Bernardini, D. Goeuriot, A. Kallel. J. Appl. Phys. 103, 054107 (2008).

[21] K. Said, G. Damamme, A. Ahmed, G. Moya, A. Kallel. Appl. Surf. Sci. 297, 45 (2014).

[22] M. Boubaya, G. Blaise. Eur. Phys. J. Appl. Phys. 37, 79 (2007).
[23] T. Thome, D. Braga, G. Blaise. J. Appl. Phys. 95, 2619 (2004).

[24] S. Le Roy, F. Baudoin, V. Griseri, G. Teyssedre. J. Appl. Phys. 112, 023704 (2012).

[25] B. Raftary, N.V. Budko, C. Voik. J. Appl. Phys. 118, 204101 (2015).

[26] R. Pacaud, T. Paulmier, P. Sarrailh. J. Appl. Phys. 122, 245106 (2017).

[27] N. Chorbel, A. Kallel, G. Damamme. Micron 112, 35 (2018).

[28] J. Liu, H.-B. Zhang, Y.-H. Ding, Z. Yan, J. Tong, Y. Yuan, Q. Zhao. Micron 16, 100 (2019).

[29] Э.И. Рау, А.А. Татаринцев, Е.Ю. Зыкова, И.П. Иваненко, С.Ю. Купреенко, К.Ф. Миннебаев, А.А. Хайдаров. ФТТ 59, 1504 (2017).

[30] E.I. Rau, A.A. Tatarintsev, E.Y. Zykova. Nucl. Instrum. Meth. Phys. Res. B 460, 141 (2019).

[31] Е.Н. Евстафьева, Э.И. Рау, А.А. Татаринцев. Вестн. МГУ. Сер. физика и астрономия 34 (2013).

[32] B. Askri, K. Raouadi, R. Renoud, B. Yangui. J. Electrostatic 67, 695 (2009).

[33] M. Kotera, K. Yamaguchi, H. Suga. Jpn J. Appl. Phys. 38, 7176 (1999).

[34] A. Palov, H. Fujii, Yu. Mankelevich, T. Rakhimova, M. Baklanov. Polymer Degradation Stability 97, 802 (2012).

[35] J.P. Vigourous, J.P. Duraud, A. Le Moel, C. Le Gressus. J. Appl. Phys. 57, 5139 (1985).

[36] G. Blaise, C. Le Gressus. AIP Adv. 8, 095228 (2018)

[37] R. Hofmann, J.R. Dennison, C.D. Thomson, J. Albertsen. IEEE Trans. Plasma Sci. 36, 2238 (2008).

[38] R. Hofmann, J.R. Dennison. IEEE Trans. Plasma Sci. 40, 298 (2012).

[39] T. Paulmier, R. Hanna, M. Belhaj et. all. IEEE Trans. Plasma Sci. 41, 3422 (2013).

[40] K. Guerch, T. Paulmier, J.R. Dennison, J. Dekany, P. Lenormand, F. Guillemet-Fritsch. J. Spacecraft Rockets 53, 1100 (2016).

[41] A.I. Titov, S.O. Kucheyev. J. Appl. Phys. 92, 5740 (2002).

[42] R. Renoud, F. Mady, C. Attard, J. Beigarre, J.-P. Ganachaud. Phys. Status Solidi A 201, 2119 (2004).

[43] S.G. Boyev, V.A. Paderin, A.P. Tyutnev. J. Electrostatics 26, 133 (1991).

[44] D.J. DiMaria, E. Cartier, D. Arnold. J. Appl. Phys. 73, 3367 (1993).

[45] В.С. Вавилов, А.С. Кив, О.Р. Ниязова. Механизмы образования и миграции дефектов в полупроводниках. Наука, М. (1981). 368 c.

\section{Редактор Ю.Э. Китаев}

